\title{
Rationale Diagnostik in der Kindergastroenterologie
}

Burkhard Rodeck

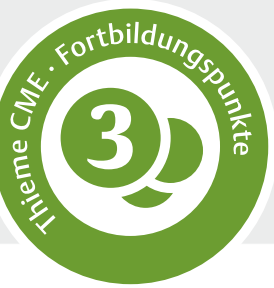

\begin{abstract}
Vom Symptom zur Diagnose - ein spannendes Thema in der Kindergastroenterologie. Da Kinder ihre Symptome meist nicht selbst oder nicht genau beschreiben können, ist es wichtig, dass Ärzte die häufigsten Symptome in den verschiedenen Altersgruppen kennen. So können sie einen sinnvollen differenzialdiagnostischen Weg entwickeln, auch um unnötige Untersuchungen zu vermeiden. Der Beitrag zeigt neben häufigen auch seltenere, in der Differenzialdiagnostik jedoch wichtige Erkrankungen.
\end{abstract}

\section{Einleitung}

Grundsätzlich lässt sich die Kindergastroenterologie in verschiedene Organerkrankungen unterteilen, nämlich in Erkrankungen des Intestinaltrakts, des Pankreas, der Leber und der ableitenden Gallenwege. Im Folgenden wird ein rationaler diagnostischer Weg für verschiedene relevante klinische Fragestellungen aufgezeigt. Dabei orientiert sich der Beitrag an der klinischen Symptomatik innerhalb der Organsysteme mit teils altersgebundenen Differenzialdiagnosen. Der Fokus liegt dabei auf den häufigen klinischen Fragestellungen bzw. den wichtigen Differenzialdiagnosen, insbesondere bei chronischen Erkrankungen und Problemen. Die Empfehlungen stützen sich auf Leitlinien, u.a. der ESPGHAN (European Society of Pediatric Gastroenterology, Hepatology and Nutrition), aktuellen Reviews zu den einzelnen Themenbereichen und meiner persönlichen Erfahrung.

\section{Erkrankungen des Intestinaltrakts bei Säuglingen und Kleinkindern mit unspezifischen Symptomen}

Das Krankheitsspektrum, das zu gastrointestinalen Symptomen (ggf. auch vermeintlich gastrointestinalen Symptomen) im Kindesalter führt, ist sehr vielgestaltig, teils altersgebunden und beschränkt sich nicht nur auf den reinen Gastrointestinaltrakt.

In den ersten Lebensjahren können Kinder naturgemäß ihre Symptome nicht selbst beschreiben. Auch Kleinkinder und junge Schulkinder sind nicht in der Lage, zwischen emotionalem und physischem Stress zu unterscheiden. Der Behandler muss daher basierend auf den Schilderungen und Interpretationen der Eltern oder Betreuungspersonen und seinem eigenen klinischen Urteil eine Entscheidung bzgl. der sinnvollen Diagnostik treffen. Bei dieser Beurteilung ist daher die Einschätzung der anamnestischen Angaben im Kontext der familiären Situation neben der klinischen Untersuchung von großer Bedeutung. Ein effizientes Management basiert auf einer vertrauensvollen therapeutischen Allianz mit den Eltern. Die folgenden klinischen Symptome führen fast immer zur elterlichen Verunsicherung und damit zu Arztvorstellungen und sind bei sonst gesunden Kindern ohne Gedeihprobleme in der Regel funktioneller Natur:

- Regurgitation

- Cyclic Vomiting Syndrome

- Kolik

- funktionelle Diarrhö (Toddler-Syndrom)

- funktionelle Obstipation

Die Verdachtsdiagnose funktioneller Probleme liegt bei bestimmten anamnestischen Voraussetzungen, einem klinisch unauffälligem Untersuchungsbefund und gutem Gedeihen und Entwicklung zwar nahe, kann allerdings erst gestellt werden, wenn die Symptome nach sorgfältiger Evaluation keiner anderen Erkrankung zugeordnet werden können.

In > Tab.1 ist die Altersgebundenheit, die teils hohe Prävalenz und der Outcome der verschiedenen Symptome dargestellt [1].

Merke

Die Anamnese trägt neben der genauen Erfassung der Symptome entscheidend zur Planung des diagnostischen Weges bei. Bei sonst gesunden Kindern erübrigt sich in den meisten Fällen eine weiterführende Diagnostik. 
- Tab. 1 Alter, Prävalenz und Outcome bei funktionellen Störungen [1].

\begin{tabular}{|c|c|c|c|}
\hline Symptom & Alter & Prävalenz in \% & Outcome \\
\hline Regurgitation & 3 Wochen bis 12 Monate & $41-67$ & verschwindet zu $90 \%$ bis zum 12 . Monat \\
\hline Rumination & 3. - 8. Monat & 1,9 & verschwindet mit der Zeit \\
\hline Cyclic Vomiting Syndrome & Säuglings- bis Jugendalter & 3,4 & $\begin{array}{l}\text { Besserungstendenz mit den Jahren, geht ggf. } \\
\text { in Migräne über }\end{array}$ \\
\hline Koliken & bis ca. 5./6. Monat & $5-19$ & verschwindet bis zum 6. Monat \\
\hline $\begin{array}{l}\text { funktionelle Diarrhö (Toddler- } \\
\text { Diarrhö) }\end{array}$ & 6. -60. Monat & $6-7$ & verschwindet meist bis zum 60. Monat \\
\hline $\begin{array}{l}\text { Dyschezie (schmerzhafte } \\
\text { Defäkation) }\end{array}$ & bis ca. 9. Monat & 2,4 & verschwindet meist bis zum 9. Monat \\
\hline Obstipation & Säuglings- bis Erwachsenenalter & $3-27$ & konsequente Therapie notwendig \\
\hline
\end{tabular}

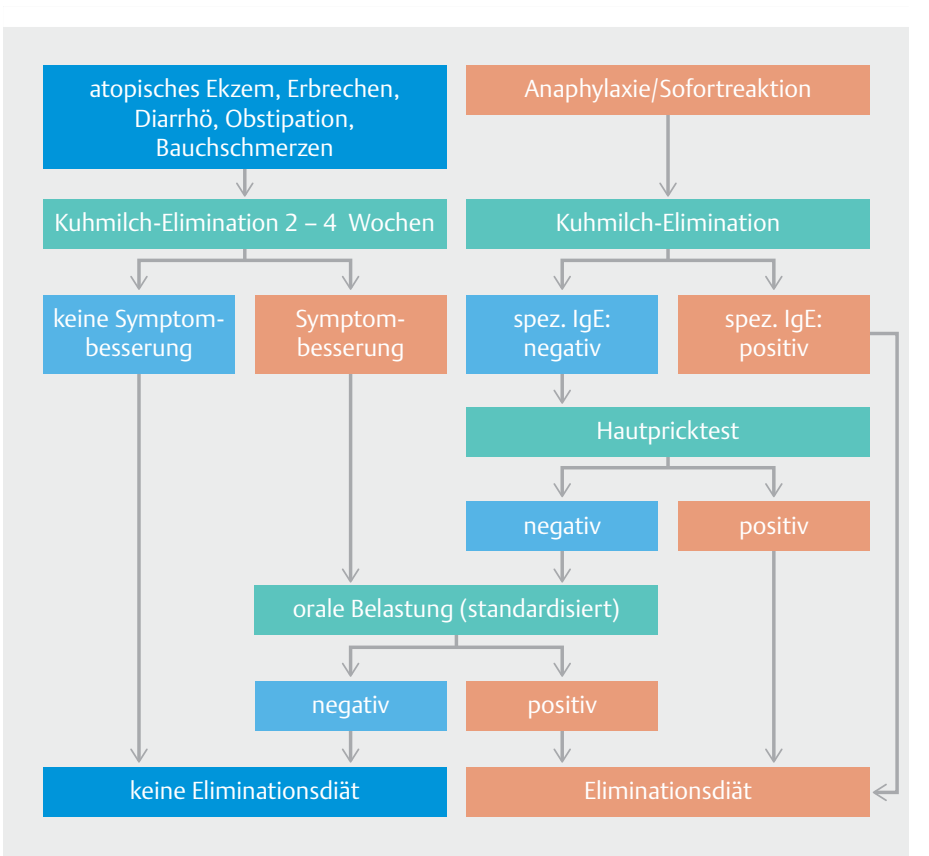

Abb. 1 Procedere bei Verdacht auf Kuhmilchproteinallergie [2].

\section{Regurgitation}

Ein gastroösophagealer Reflux im Säuglings- und jungen Kleinkindalter ist physiologisch, bis zum Ende des 1. Lebensjahres reift der gastroösophageale Verschlussmechanismus in der Regel aus, so dass grundsätzlich kein Handlungsbedarf besteht. Steigt das Refluxat über den Ösophagus hinaus, also spuckt das Kind, spricht man von Regurgitation.

Eine Refluxerkrankung liegt vor, wenn eine Ösophagitis (mit retrosternalen Schmerzen) vorliegt, eine Gedeihstörung zu beobachten ist oder respiratorische Probleme (meist obstruktiver Art) bei rezidivierenden Mikroaspirationen auftreten.
Merke

Eine unkritische probatorische Therapie mit Säureblockern bei Verdacht auf einen gastroösophagealen Reflux im Säuglingsalter ist nicht sinnvoll.

Eine gastroösophageale Refluxerkrankung kann in der Folge einer Kuhmilchallergie auftreten, so dass eine probatorische Umstellung auf eine hypoallergene Therapiemilch als diagnostische Eliminationsdiät über ca. 2-4 Wochen sinnvoll ist. Dabei sollte ein Symptomprotokoll geführt werden, danach erfolgt wieder Kuhmilchformula-Belastung. Nur, wenn es nach initialer Besserung wieder vermehrt zu Problemen unter der Belastung kommt, kann die Diagnose einer Kuhmilchproteinallergie gestellt werden [2]. \Abb. 1 zeigt das Vorgehen der diagnostischen Einschätzung im Säuglings- und Kleinkindalter. Kürzlich wurde ein Score-System zur Erkennung von Kuhmilchproteinallergien vorgestellt ( $\triangleright$ Tab.2), das zwar streng wissenschaftlich noch nicht evaluiert ist, im täglichen Gebrauch im Sinne eines Screenings jedoch sinnvoll erscheint [3]. Es ersetzt nicht die Notwendigkeit einer gezielten Elimination mit anschließender Belastung.

In einer Metaanalyse effektiver Behandlungsstrategien von Koliken bei gestillten Kindern konnte gezeigt werden, dass eine Behandlung mit Probiotika (insbesondere Lactobazillus reuteri) oder Fenchelpräparaten erfolgreicher als eine Placebobehandlung sein kann [4].

Merke

Bei „Dreimonatskoliken“ kann eine diagnostische Eliminationsdiät mit hypoallergener Therapiemilchformula zur Sicherung der Verdachtsdiagnose Kuhmilchproteinallergie sinnvoll sein.

Die diagnostisch zur Verfügung stehenden Möglichkeiten haben alle Grenzen. Die pH-Metrie detektiert nur saure Refluxepisoden, eine Ösophagogastroduo- 
\Tab. 2 CoMiSS Cows Milk-related Symptom Score [3]. In der Spalte „Score“ werden dann die individuellen Punktzahlen eingetragen und addiert.

\begin{tabular}{|c|c|c|c|}
\hline Symptom & Punkte & Beschreibung & Score \\
\hline \multirow[t]{7}{*}{ Schreien (seit mind. 1 Woche) } & 0 & $<1 \mathrm{~h} /$ Tag & \\
\hline & 1 & $1-1,5 \mathrm{~h} / \mathrm{Tag}$ & \\
\hline & 2 & $1,5-2 \mathrm{~h} / \mathrm{Tag}$ & \\
\hline & 3 & $2-3 \mathrm{~h} / \mathrm{Tag}$ & \\
\hline & 4 & $3-4 h / T a g$ & \\
\hline & 5 & $4-5 \mathrm{~h} / \mathrm{Tag}$ & \\
\hline & 6 & $>5 \mathrm{~h} / \mathrm{Tag}$ & \\
\hline \multirow[t]{7}{*}{ Spucken } & 0 & 0 -bis 1 -mal/Tag & \\
\hline & 1 & $\geq 3-$ bis $\leq 5-\mathrm{mal} / \mathrm{Tag}$ & \\
\hline & 2 & >5-mal>1 Kaffeelöffel & \\
\hline & 3 & $>5$-mal \pm halbe Trinkmenge bei weniger als der Hälfte der Mahlzeiten & \\
\hline & 4 & ständiges Spucken kleiner Mengen > 30 min nach jeder Mahlzeit & \\
\hline & 5 & Spucken > als halbe Mahlzeit bei mind. der Hälfte der Mahlzeiten & \\
\hline & 6 & Spucken der kompletten Mahlzeit nach jeder Mahlzeit & \\
\hline \multirow[t]{5}{*}{ Stühle (Bristol-Skala) } & 4 & harte Stühle (Typ 1 und 2) & \\
\hline & 0 & normale Stühle (Typ 3 und 4) & \\
\hline & 2 & weiche Stühle (Typ 5) & \\
\hline & 4 & $\begin{array}{l}\text { flüssige Stühle (Typ 6) } \\
\text { keine Infektion }\end{array}$ & \\
\hline & 6 & wässrige Stühle (Typ 7) & \\
\hline \multirow[t]{2}{*}{ Hautsymptome } & $0-6$ & $\begin{array}{l}\text { atopisches Ekzem: } \\
\begin{array}{l}\text { " Kopf-Hals-Rumpf: } \\
-0 \text { nicht vorhanden } \\
-1 \text { leicht } \\
-2 \text { mittelschwer } \\
-3 \text { schwer } \\
\text { - Arme-Hände-Beine-Füße: } \\
-0 \text { nicht vorhanden } \\
-1 \text { leicht } \\
-2 \text { mittelschwer } \\
-3 \text { schwer }\end{array}\end{array}$ & \\
\hline & 0 oder 6 & $\begin{array}{l}\text { Urtikaria: } \\
\text { - } 0 \text { nein } \\
\text { - } 6 \text { ja }\end{array}$ & \\
\hline \multirow[t]{5}{*}{ Atemwegssymptome } & 0 & keine & \\
\hline & 1 & geringe & \\
\hline & 2 & leichte & \\
\hline & 3 & schwere & \\
\hline & & Gesamtscore: & \\
\hline
\end{tabular}


denoskopie kann zwar eine Ösophagitis und andere Pathologien im oberen Gastrointestinaltrakt erkennen, bei unauffälligem Befund können aber refluxbedingte Beschwerden nicht ausgeschlossen werden. Das Ausmaß der Beschwerden korreliert nicht gut mit dem endoskopisch-histologischem Befund. Zukünftig wird die kombinierte $\mathrm{pH}$-Metrie mit ösophagealer Impedanzmessung der Goldstandard in der Refluxdiagnostik werden. Damit können auch nicht saure Refluxepisoden quantifiziert werden. Die Methode ist allerdings zeitaufwändig, Normwerte im Kindesalter werden zurzeit evaluiert, die Technik steht noch nicht flächendeckend zur Verfügung.

Eine Sonografie bei einem spuckenden Kind bezüglich Reflux ist verzichtbar, das Spucken (Regurgitation) allein beantwortet die Frage-eine Quantifizierung der Refluxepisoden ist mit der Sonografie kaum standardisiert möglich. In der Differenzialdiagnose kann sie allerdings zur Erkennung von Passagestörungen im Magen-/Darmtrakt beitragen.

\section{Cyclic Vomiting Syndrome}

Zyklisches Erbrechen gehört zu den funktionellen Störungen des Gastrointestinaltrakts. Typischerweise kommt es zu transienten (wenige Stunden bis zu 10 Tagen) individuell stereotypen Attacken mindestens zweimal in 6 Monaten mit Übelkeit und anfallsartigem Erbrechen (mindestens $4 / \mathrm{h}$ über mindestens 1 Stunde), häufig in der 2. Nachhälfte. Zwischen den Episoden sind die Kinder gesund und beschwerdefrei. Abgesehen von der typischen Anamnese und fehlenden Hinweisen auf andere Erkrankungen gibt es keinen diagnostischen Marker. Die funktionelle Störung tritt nicht nur im Säuglingsalter auf.

\section{Kolik}

Im Säuglingsalter ist einer der häufigsten Vorstellungsgründe das unruhige, schreiende Kind, das im jungen Säuglingsalter unter der Verdachtsdiagnose „Dreimonatskolik“ in eine oft somatisch orientierte Diagnostikschiene fällt, die hinterfragt werden muss. Meist steht eine Regulationsstörung dahinter, immer wieder wird aber auch ein gastroösophagealer Reflux oder eine Nahrungsmittelunverträglichkeit vermutet. Säuglinge mit Regulationsstörungen haben Schwierigkeiten, ihr Verhalten in regulativen und Interaktionskontexten adäquat zu steuern.

Koliken werden zu den funktionellen Störungen gezählt. Nach den Rome-III-Kriterien sind sie durch anfallsartiges Schreien oder Unruhe ohne klar erkennbare Ursache von Beginn und Ende über mindestens 3 Stunden am Tag, mindestens 3 Tage in der Woche, mindestens über 1 Woche bei sonst gesunden, gut gediehenen Säuglingen gekennzeichnet. Die Anamnese führt zur diagnostischen Einschätzung.

\section{Funktionelle Dyspepsie (Toddler-Diarrhö)}

Mit einer Prävalenz von bis zu 7\% ist die funktionelle Dyspepsie im Kleinkindalter nicht selten. Den klinisch sonst vollkommen gesund wirkenden Kindern wird oft der Kindergartenbesuch unter Hinweis auf eine mögliche infektiöse Genese verweigert. Die Kinder selbst beklagen keinerlei Symptome. Typischerweise haben die Kinder bis zu 5-6 lockere Stühle am Tag, teils auch mit unverdauten Nahrungsmittelbestandteilen, nachts in der Regel kein Stuhlgang. Differenzialdiagnostisch können folgende Erkrankungen erwogen werden (Handlungsempfehlungen in Klammern):

- Postenteritis-Syndrom (probatorisch milchfreie Diät über 4 Wochen)

- gastrointestinale Infektion (u. a. Giardia lamblia, Cryptosporidien im Stuhl)

- Fruktose-Malabsorption (probatorisch fruktosereduzierte Kost)

- Zöliakie (siehe dort)

- Obstipation mit Überlaufenkopresis (Sonografie, klinische Untersuchung)

\section{PRAXISTIPP}

Diagnostik zum Ausschluss von Organerkrankungen bei funktioneller Dyspepsie

Blutbild, CRP, BSG, Transglutaminase-Antikörper, IgA, Stuhlkultur (Clostridium difficile, Giardia lamblia), Calprotectin im Stuhl (Cave: altersabhängige Normwerte), ggf. IgE und spezifisches Nahrungsmittel-IgE (Fx5), um seltene IgE-vermittelte Nahrungsmittelsensibilisierungen aufzuspüren.

\section{Funktionelle Obstipation}

Diese funktionelle Störung, die nicht nur im Säuglingsalter auftritt, ist weiter unten dargestellt.

\section{Erkrankungen des Intestinaltrakts - altersunabhängige Symptome}

\section{Chronischer Durchfall}

\section{Kongenitale Diarrhö}

Ursache einer kongenitalen Diarrhö sind seltene und unterschiedliche, meist angeborene malabsorptive oder sekretorische Erkrankungen. Sie manifestieren sich in der Regel in den ersten Lebenswochen mit massiven Durchfällen mit nachfolgender Dehydratation und sind schwierig zu therapieren (intractable diarrhea). Klinisch unterscheidet man eine osmotische, eine sekretorische und eine inflammatorische Diarrhö. Liegt eine Störung mit Malabsorption oder eine osmotische Diarrhö vor, bessert sich der Durchfall 


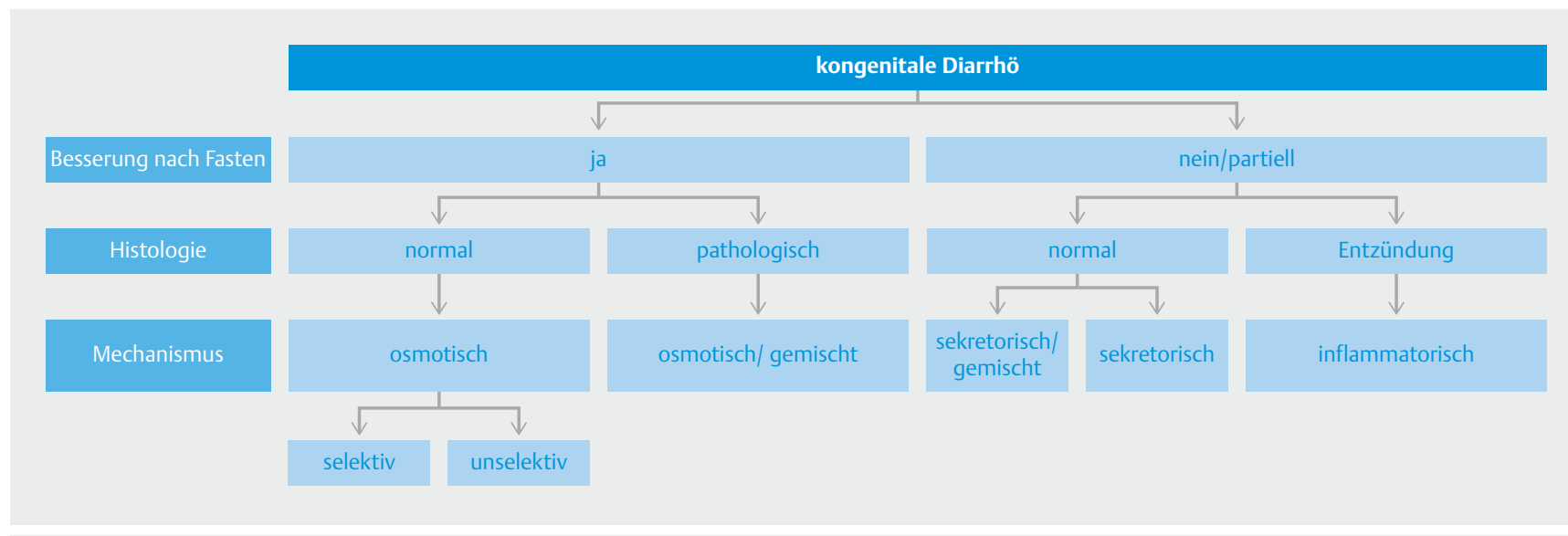

Abb. 2 Einteilung kongenitale Diarrhö [5].

> Tab.3 Differenzialdiagnose kongenitaler Diarrhö (Quelle [5]).

\begin{tabular}{|c|c|c|}
\hline Typ & Differenzialdiagnose & \\
\hline Osmotische Diarrhö & $\begin{array}{l}\text { selektiv: } \\
\text { Kongenitaler Laktasemangel } \\
\text { Sacharase-Isomaltasemangel } \\
\text { Maltase-Glukoamylasemangel } \\
\text { Enteropeptidasemangel } \\
\text { Glukose-Galaktose-Malabsorption } \\
\text { Lysinurische Proteinintoleranz } \\
\text { Prohormon-Convertasemangel }\end{array}$ & $\begin{array}{l}\text { unselektiv: } \\
\text { Mukoviszidose } \\
\text { Hereditäre Pankreatitis } \\
\text { Lipasemangel } \\
\text { Chylomikronen-Retentionserkrankung } \\
\text { Hypobetalipoproteinämie } \\
\text { Abetalipoproteinämie } \\
\text { Shwachman-Diamond-Syndrom }\end{array}$ \\
\hline $\begin{array}{l}\text { Osmotisch/ } \\
\text { gemischte Diarrhö }\end{array}$ & $\begin{array}{l}\text { Nahrungsmittelallergie } \\
\text { Intestinale Lymphangiektasie } \\
\text { Microvillus-Inklusionserkrankung } \\
\text { Intestinale Epitheldysplasie } \\
\text { Autoimmun-Polyendokrinopathie-Candidiasis- } \\
\text { ektodermale Dysplasie }\end{array}$ & \\
\hline $\begin{array}{l}\text { Sekretorisch/ } \\
\text { Gemischte Diarrhö }\end{array}$ & Acrodermatitis enteropatica & \\
\hline $\begin{array}{l}\text { Inflammatorische } \\
\text { Diarrrhö }\end{array}$ & $\begin{array}{l}\text { Enteritis } \\
\text { IPEX, IPEX-like } \\
\text { Ektodermale Dysplasie-Formen } \\
\text { Interleukindefekte } \\
\text { Chronisch granulomatöse Erkrankungen } \\
\text { Leukozytenadhäsionsdefekte }\end{array}$ & $\begin{array}{l}\text { Glykogenspeichererkrankung } 1 \mathrm{~b} \\
\text { SCID } \\
\text { Wiskott-Aldrich-Syndrom } \\
\text { X-linked Agammaglobulinämie } \\
\text { IgA-Mangel } \\
\text { CVID } \\
\text { X-linked lymphoproliferatives Syndrom } \\
\text { u. a. }\end{array}$ \\
\hline
\end{tabular}

beim Fasten. Bei sekretorischer oder entzündlicher Genese ändert sich die klinische Symptomatik nicht. Die Diagnostik beruht daher zunächst auf einem Fastentest, im 2.Schritt auf einer Histologie der Darmschleimhaut. Damit können morphologisch-strukturelle Veränderungen der Schleimhaut wie die Zottenstruktur, die Verteilung der Enterozyten und entzündliche Aktivität dargestellt werden. Wichtige anamnestische Fragen sind:

- Polyhydramnion

- Konsanguinität der Eltern

- Beginn der Symptomatik mit der Nahrungsaufnahme
- Abb. $\mathbf{2}$ zeigt den Typ, > Tab. $\mathbf{3}$ die entsprechenden Differenzialdiagnosen der Diarrhö.

Pathophysiologisch können $\mathbf{4}$ Gruppen unterschieden werden [5]:

- Störungen der Digestion, Absorption, des Transports von Nährstoffen oder Elektrolyten

- Störungen der Differenzierung oder Polarisierung der Enterozyten

- Störungen der enteroendokrinen Zellen

- Immundefekte 


\section{Zuckermalabsorptionen}

Erster Hinweis für das Vorliegen einer Zuckermalabsorption ist die Ernährungsanamnese.

\section{Laktoseintoleranz}

Eine primäre Laktoseintoleranz (adulte Hypolaktasie) kann sich bei nachlassender Laktaseaktivität in den Enterozyten entwickeln.

Merke

Mit zunehmendem Alter lässt die Laktaseaktivität nach, so dass die intakte Laktose in tiefere Darmabschnitte gelangen kann und dort zu Methan, Laktat und Wasserstoff verstoffwechselt wird, was zu Gasbildung, lockeren Stühlen und Schmerzen führen kann.

Vor ca. 10000 Jahren haben sich bei Populationen, die Milchwirtschaft betrieben haben, Mutationen entwickelt, die zu einer Persistenz der Laktaseaktivität auch im Erwachsenenalter führen. Man geht davon aus, dass etwa 85-90\% der Bevölkerung in Deutschland diese Mutation tragen, so dass sie Milchzucker auch in höherem Alter gut vertragen. Die Mutationen betreffen den Promotorbereich des Laktasegens auf dem langen Arm des Chromosoms 2 (2q21): C/T13910-Polymorphismus oder G/A-22018-Polymorphismus. In Deutschland sind ca. 10-15\% der Bevölkerung von der Laktoseintoleranz betroffen, wobei die Restaktivität der Laktase und damit die Symptomatologie unterschiedlich sein können.

Säuglinge und junge Kleinkinder sind nur in den ganz seltenen Fällen einer angeborenen Alaktasie von einer primären Laktoseintoleranz betroffen, eine leider häufig geratene probatorische milchzuckerfreie Kost in diesem Alter ist daher sinnlos. Die Klinik der adulten Hypolaktasie beginnt allenfalls im späten Kleinkindalter, meist erst bei Jugendlichen oder jungen Erwachsenen.

Zu berücksichtigen sind allerdings sekundäre Formen der Laktoseintoleranz, die bei allen Erkrankungen mit morphologischen Störungen der Darmschleimhaut auftreten können (Zöliakie, Nahrungsmittelallergien, chronisch entzündliche Darmerkrankungen, protrahierte Gastroenteritis, etc.). Diagnostisch kann ein Auslassversuch (Laktoseelimination in der Ernährung) über 4 Wochen genutzt werden mit Verschwinden der Symptome, oder ein Wasserstoffexhalationstest $\left(\mathrm{H}_{2}\right.$ Atemtest). Die genetische Diagnostik ( $\mathrm{T} / \mathrm{C}$ - und $\mathrm{A} / \mathrm{G}$ Polymorphismus im Laktase-Gen) sagt bei der unterschiedlichen Restaktivität der Laktose kaum etwas über den Zusammenhang von Bauchschmerzen und Laktoseintoleranz aus. Der Laktosebelastungstest mit Gabe von Laktose und anschließenden Blutzuckerkontrollen spielt im Kindesalter keine Rolle.

\section{Merke}

Eine primäre Laktoseintoleranz im Säuglings- und jungen Kleinkindalter liegt praktisch nie vor. Die molekulargenetische Diagnostik korreliert nicht unbedingt mit den klinischen Symptomen.

\section{Fruktosemalabsorption}

Viele Kinder trinken zur Durstlöschung heute nicht mehr nur Wasser, sondern Saftschorlen, Fruchtsäfte oder Fertiggetränke. In einigen Früchten ist der Fruktosegehalt deutlich höher als der Glukosegehalt (z. B. bei Apfel, Birne, Mango oder Wassermelone).

\section{Merke}

Fruktose wird nahezu äquimolar zu Glukose aufgenommen. Zudem ist die individuelle Aufnahmekapazität des Dünndarms unterschiedlich, so dass bei vielen Kindern Fruktose nur partiell absorbiert wird.

Die verbleibenden Fruktosemoleküle gelangen in den Dickdarm und werden von der dortigen bakteriellen Flora vergoren. Das führt zu Bildung von Gas (Wasserstoff, Methan) und von osmotisch wirksamen Substanzen, wie Fettsäuren. Damit erklären sich klinische Probleme wie Meteorismus, Bauchschmerzen und Durchfall. Wie bei der Laktoseintoleranz kann die Diagnose durch probatorische Fruktosereduktion in der Ernährung über 4 Wochen (und Besserung der Beschwerden) oder durch einen $\mathrm{H}_{2}$-Atemtest gestellt werden.

\section{Zuckerersatzstoffmalabsorption (Sorbit, Xylit)}

Zuckerersatzstoffe werden von der Nahrungsmittelindustrie zum Süßen von Getränken (z. B. Eistee) und Genussmitteln (z. B. Kaugummi) eingesetzt. Auch hier ist die individuelle Aufnahmekapazität des Darms begrenzt, so dass bei entsprechend höherem Genuss klinische Probleme wie Bauchschmerzen und Durchfall auftreten können. Sorbit ist auch normaler Bestandteil von verschiedenen Nahrungsmitteln (z.B. Trockenfrüchten, Frucht der Eberesche, Birnen, Pflaumen, Äpfeln, Aprikosen, Pfirsichen und Rosinen). Diagnostisch kann ein Auslassversuch weiterführen oder ein $\mathrm{H}_{2}$ Atemtest.

\section{Merke \\ Beim Apfelschorletrinker, Eisteetrinker oder Kau- gummikauer mit Bauchschmerzen, Blähungen und/ oder lockeren Stühlen liegt häufig eine Fruktose-/ Sorbitmalabsorption vor.}

\section{Chronisch entzündliche Darmerkrankungen}

Chronisch entzündliche Darmerkrankungen (CED) sind rekurrierende systemische inflammatorische Erkrankungen, die sich primär im Magen-Darm-Trakt manifestieren. Als Grund wird eine Störung der intestinalen Homöostase (Erkennung und Abwehr intestinaler Pathogene, Toleranz der eigenen Darmflora und Nah- 


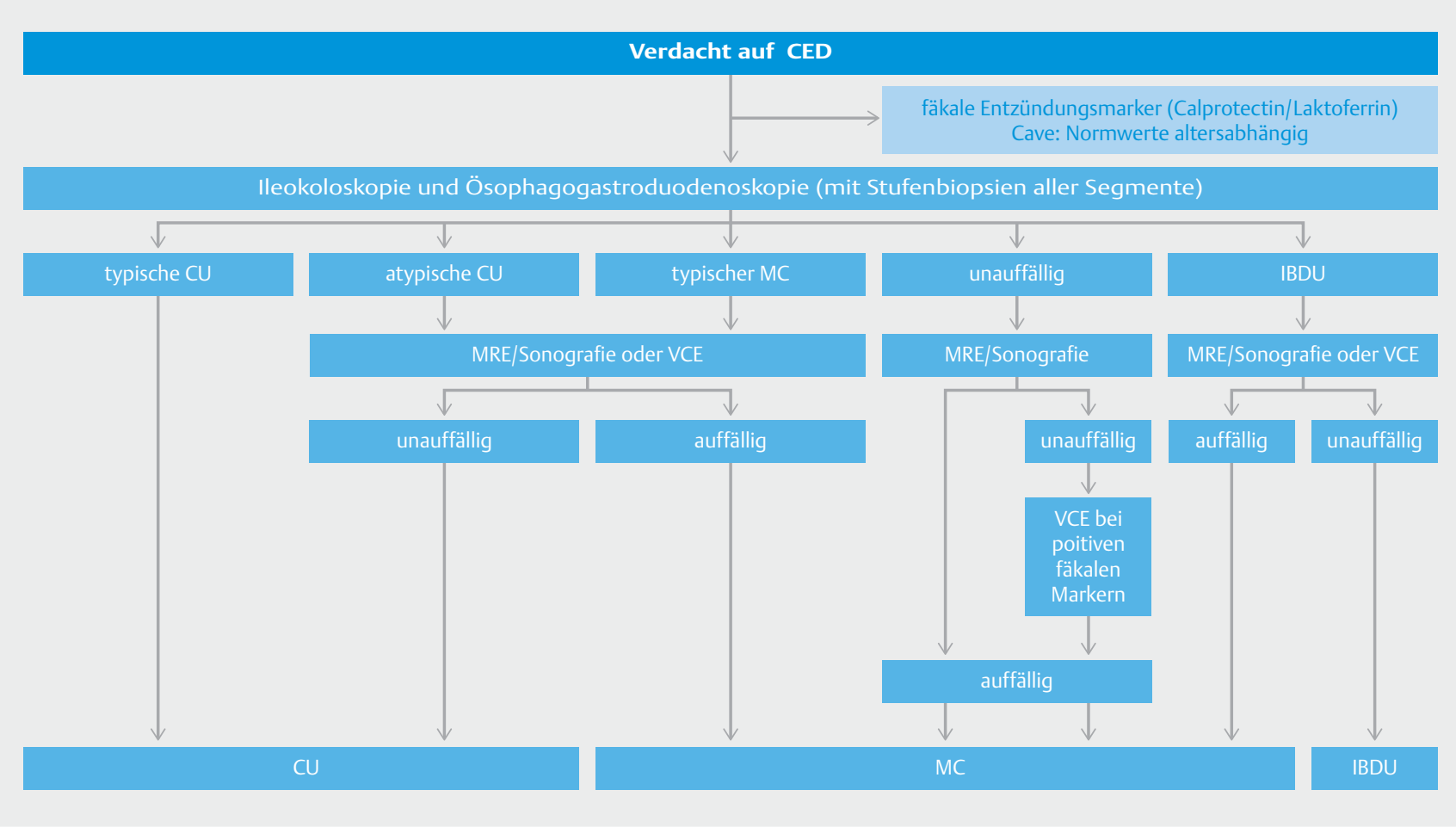

Abb. 3 Diagnostik bei V. a. chronisch entzündliche Darmerkrankung (CED). CU: Colitis ulcerosa, MC: M. Crohn, IBDU: nicht klassifizierbare CED, MRE: Magnetresonanzenterografie, VCE: Videokapselendoskopie.

rungsantigene) auf dem Boden einer genetischen Suszeptibilität und Einfluss von Umweltfaktoren angenommen. Man unterscheidet 3 Typen der CED:

- Morbus Crohn

- Colitis ulcerosa

- eine nicht klassifizierte Form (inflammatory bowel disease unclassified, IBDU), die sich in der primären Organmanifestation und dem Verlauf unterscheiden

Insbesondere Morbus Crohn und Colitis ulcerosa können zu einer deutlichen körperlichen und psychosozialen Beeinträchtigung führen mit erheblichen Einschränkungen von Lebensqualität und im späteren Leben beruflicher Integration und Belastbarkeit.

Die typischen Zeichen einer CED sind:

- Bauchschmerzen

- Durchfall

- Gewichtsverlust

- Leistungsabfall

- ggf. Fieber

- Anämie

- extraintestinale Manifestationen

Bei einer Colitis ulcerosa sind die Zeichen der Dickdarmentzündung wie blutig-schleimige Durchfälle häufiger. Die Erkrankungen verlaufen rekurrierend in Schüben.
Die diagnostischen Kriterien einer CED sind von einer Arbeitsgruppe der ESPGHAN als sog. Portokriterien definiert und 2014 aktualisiert worden [6].

Die Diagnose basiert auf anamnestischen und klinischen Befunden, Laboruntersuchungen, Endoskopie (Ösophagogastroduodenoskopie, Ileokoloskopie mit Histologie) und einer bildgebenden Diagnostik des Dünndarms (Sonografie, Magnetresonanzenterografie, Videokapselendoskopie). Infektiöse Ursachen müssen ausgeschlossen werden (Stuhlkultur, Clostidien, CMVDiagnostik).

In > Abb. $\mathbf{3}$ ist der diagnostische Workup bei CED dargestellt. Bei Kleinkindern und Säuglingen können Crohn-like Diseases Hinweise auf einen zugrundeliegenden Immundefekt sein, so dass in dieser Altersgruppe auch eine immunologische Diagnostik angezeigt ist.

Im Verlauf der Erkrankung werden die Krankheitsaktivität und damit das Ansprechen auf die Therapie mit Aktivitäts-Scores überprüft, die zwar nicht zur Initialdiagnostik entwickelt wurden, deren einzelne Items aber Hinweise für die Diagnose bieten. Beim Morbus Crohn wird der Pediatric Crohns Disease Activity Index (PCDAI) verwendet, bei der Colitis ulcerosa der Pediatric Ulcerative Colitis Activity Index (PUCAI). 


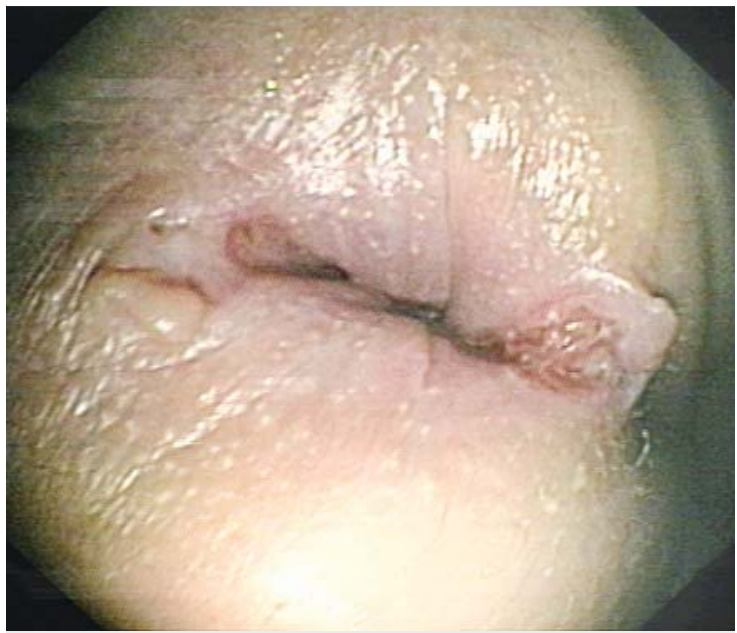

Abb. 4 Perianale Läsionen bei Morbus Crohn.

Der PCDAI umfasst anamnestische Parameter (Bauchschmerzen, Stuhlparameter, generelles Befinden), klinische Parameter (Gewicht, Größe, abdomineller Befund, perianale Symptome, extraintestinale Manifestationen) und Laborparameter (Hämatokrit, Blutsenkungsgeschwindigkeit, Albumin). In der Initialdiagnostik sind weitere Inflammationsparameter wegweisend, wie CRP und auch Thrombozytose. Bei der klinischen Untersuchung muss unbedingt auch eine rektale/anale Inspektion erfolgen. Marisken, Fissuren, Fisteln oder auch ein perianales Exanthem sind starke Hinweise für Morbus Crohn (> Abb.4).

Der PUCAI berücksichtigt anamnestische und klinische Parameter wie Bauchschmerzen, rektale Blutung, Stuhlkonsistenz und -frequenz, nächtlicher Stuhlgang sowie den Aktivitätslevel. Bei einer Colitis ulcerosa sind die systemischen Inflammationsparameter in der Regel weniger betroffen als beim Morbus Crohn. Bei allen Formen der CED sind die fäkalen Inflammationsmarker wie Calprotektin und Laktoferrin aus der Diagnostik nicht mehr wegzudenken. Bei der Beurteilung ist auf den altersabhängigen Referenzbereich zu achten. Die fäkalen Marker sind auch bei akuten Gastroenteritiden (bei bakterieller Ursache mehr als bei Virusinfektion), bei Nahrungsmittelallergien, Zöliakie oder Immundefekten erhöht, so dass diese Erkrankungen in der Differenzialdiagnose zu erwägen sind.

Merke

Bei Verdacht auf eine chronisch entzündliche Darmerkrankung muss immer eine rektale Inspektion durchgeführt werden. Zur ersten Screeningdiagnostik gehören die fäkalen Inflammationsmarker (Calprotektin oder Laktoferrin).

\section{Nahrungsmittelallergie}

Die Grundsätze der Diagnostik bei Nahrungsmittelallergien entsprechen denen bei Kuhmilchallergien. Nahrungsmittelallergien, die mit unspezifischen Symptomen wie Bauchschmerzen einhergehen, sind meist nicht IgE-vermittelt und damit mit der IgE-gestützten Diagnostik nicht erkennbar. In diesen Fällen muss das vermutete Allergen eliminiert werden, sich die Symptomatik protokolldokumentiert bessern und nach Belastung ebenfalls dokumentiert wieder verschlechtern.

Zöliakie

Eine Zöliakie ist eine immunmediierte Systemerkrankung, induziert durch das mit der Nahrung aufgenommene Gluten und verwandte Prolamine bei genetischer Veranlagung. Die klinische Manifestation ist unterschiedlich und kann sich auch monosymptomatisch/ oligosymptomatisch ohne eine typische Enteropathie mit Bauchschmerzen, Durchfall und Gedeihstörung präsentieren.

Merke

Das klassische klinische Bild mit Gedeihstörung, Durchfall und Buchschmerzen liegt bei einer Zöliakie heute seltener vor. Meist sind es monosymptomatische oder oligosymptomatische Verlaufsformen.

Als erstes werden die Transglutaminase-Antikörper (IgA-anti-TG2) und das Gesamt-IgA bestimmt. Bei einem IgA-Mangel sind die IgA-anti-TG2 nur mit Einschränkungen bewertbar. Sind die IgA-anti-TG2 mehr als das 10-fache der Norm erhöht, erfolgt eine Kontrolle der Endomysiumantikörper (EMA) und eine Untersuchung der HLA-Haplotypen DQ2 und DQ8. Bei passender Klinik, positiven EMA und HLA-DQ2-und/oder HLA-DQ8-Positivität kann die Diagnose einer Zöliakie ohne Endoskopie gestellt werden. Die HLA-Loci DQ2 oder DQ8 werden allerdings auch bei der gesunden Normalbevölkerung bei ca. $30 \%$ gefunden, so dass sie im positiven Fall nur in Kombination mit den anderen Parametern genutzt werden können. Im Zweifel muss eine Ösophagogastroduodenoskopie mit Gewinnung von Duodenalbiopsien durchgeführt werden. Die histologische Einteilung erfolgt nach den MARSH-Kriterien.

Merke

Erst nach Sicherung der Diagnose darf mit einer glutenfreien Kost begonnen werden.

PRAXISTIPP

Labordiagnostik bei Verdacht auf Zöliakie

IgA, Transglutaminase-Antikörper (IgA-antiTG2), Endomysium-Ak, HLA-DQ2 /HLA DQ8 


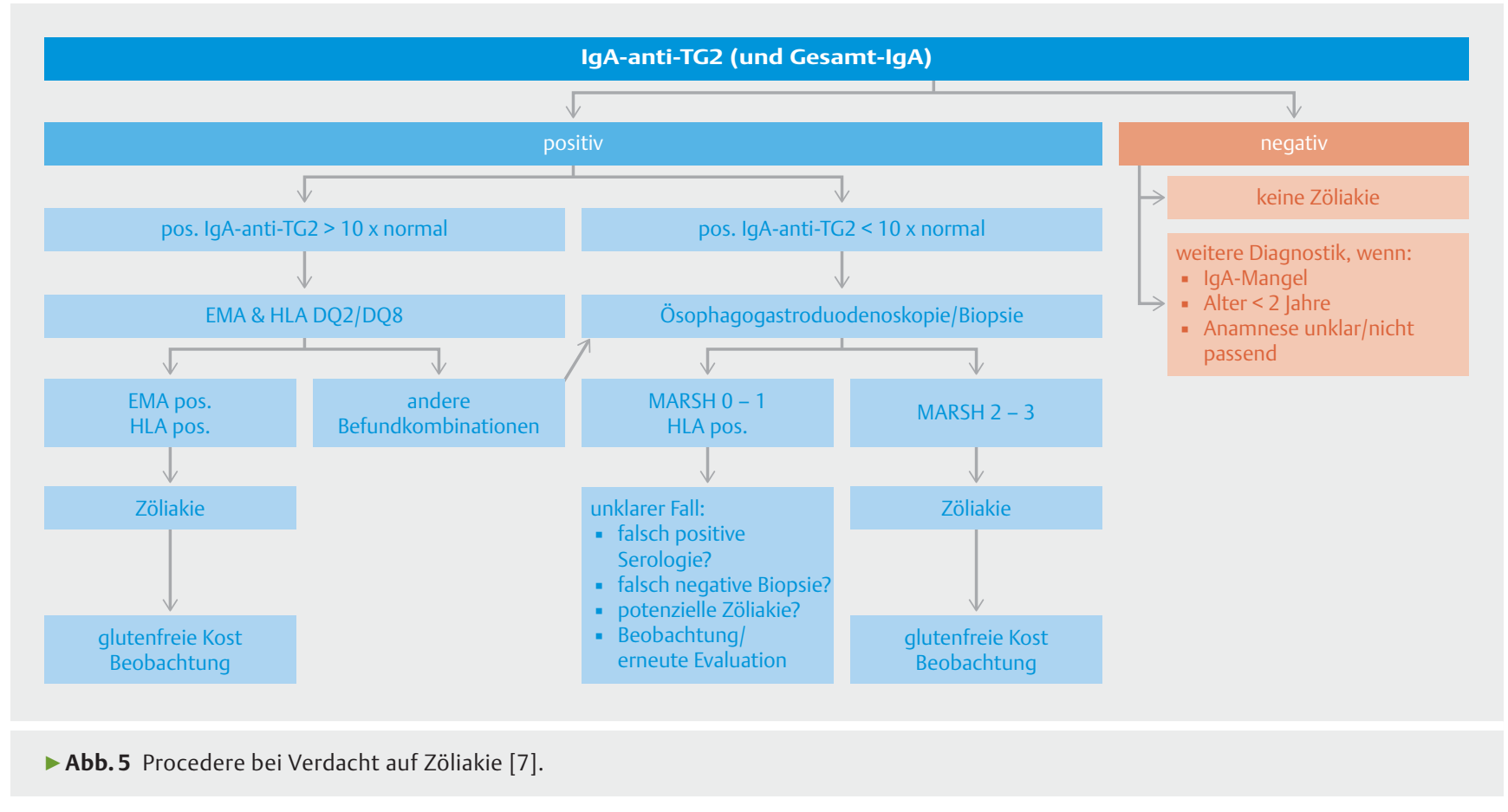

- Abb. 5 stellt den von der ESPGHAN empfohlenen Algorithmus zur Diagnostik einer Zöliakie dar [7].

\section{Obstipation}

Ca. 25\% der Vorstellungen bei Kindergastroenterologen erfolgen aufgrund einer Verstopfung. Damit ist es auch in der pädiatrischen Praxis ein relevantes Problem. Nur in wenigen Fällen liegt eine organische Erkrankung vor. Eine gründliche Anamnese ist auch hier für die richtige Weichenstellung wichtig.

Wichtige Informationen sind:

- verspäteter Mekoniumabgang

- Beginn und Dauer der Symptomatik

- Ernährungsanamnese

- Medikamentenanamnese

- Stuhlanamnese (Häufigkeit, Konsistenz, Portionsgröße, Defäkationsschmerz, Hämatochezie, Stuhlinkontinenz)

- Bauchschmerzen

Das häufig anzutreffende typische Stuhlvermeidungsverhalten spricht für einen funktionellen Charakter. Kinder scheuen oft den Toilettengang wegen der Unattraktivität/Verschmutzung der zur Verfügung stehenden Toiletten in öffentlichen Bereichen (Kindergarten, Schule, etc.). Auf eine organische Ursache können hinweisen:

- Fieber

- ausladendes Abdomen

- Anorexie

- Gewichtsverlust

- mangelndes Gedeihen
In diesen Fällen sollte schon früh eine organorientierte Diagnostik erfolgen.

\section{Merke}

Bei Kindern mit statomotorischen/psychomotorischen Entwicklungsstörungen oder immobilen Kindern ist die Obstipation ein sehr häufiges Problem.

Organische Ursachen einer Obstipation sind:

- anatomische Ursachen (Analstenose, ventral verlagerter Anus, Tumor im kleinen Becken)

- metabolische und gastrointestinale Ursachen (Hypothyreose, Hyperkalzämie, Hypokaliämie, Mukoviszidose, Diabetes mellitus, Zöliakie, Kuhmilchproteinallergie)

- Neuropathien (Rückenmarkserkrankungen, Tethered Cord, Neurofibromatose, Enzephalopathien)

- intestinale nervale oder muskuläre Erkrankungen (Morbus Hirschsprung, viszerale Myopathien oder Neuropathien)

- abdominelle Muskelerkrankungen (Prune-BellySyndrom, Gastroschisis)

- Connective Tissue Disorders (Sklerodermie, systemischer Lupus erythematosus, Ehlers-DanlosSyndrom)

- Medikamente (Opiate, Phenobarbital, SucralfatAntacida, Antihypertensiva, Anticholinergika, Antidepressiva, Sympathomimetica)

Bei vielen der Erkrankungen ist die Obstipation nur ein Nebensymptom, sie sind durch die Hauptsymptome leicht erkennbar. Die klinische Untersuchung umfasst 


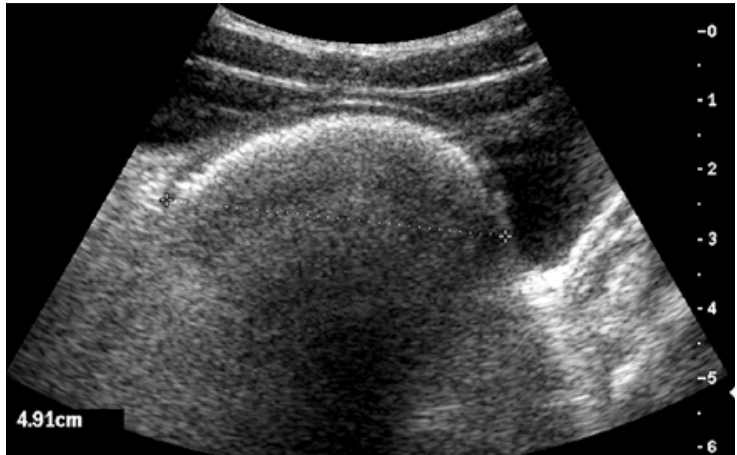

Abb. 6 Rektale Stuhlimpaktion bei Obstipation (Unterbauchquerschnitt).

immer eine anale Inspektion (wenn möglich mit rektaldigitaler Untersuchung) und eine neurologische Untersuchung (u. a. Analreflex). Eine abdominelle Sonografie sollte immer erfolgen, in der Regel ist die rektale Stuhlimpaktion (Rektumweite $>3 \mathrm{~cm}$ ) eindrucksvoll zu sehen ( Abb. 6).

Wenn es aufgrund der Anamnese und der klinischen Untersuchung keine Hinweise auf eine übergeordnete Erkrankung oder einen Morbus Hirschsprung gibt, kann zunächst ein Therapieversuch mittels Macrogolpräparaten erfolgen. Bei Misserfolg nach ca. 6 Monaten sollte dann eine erweiterte Diagnostik stattfinden.

Die organorientierte Diagnostik umfasst entsprechend TSH, Transglutaminase-Antikörper (IgA-anti-TG2), IgA, Elektrolyte. Bei Verdacht auf Morbus Hirschsprung (später Mekoniumabgang, leere, enge Ampulle, Unmöglichkeit, großvolumige Stühle anzusetzen) muss eine Rektumsaugbiopsie zur neuropathologischen Untersuchung (Ganglienzellen, Acetylcholinesterase-Aktivität) gewonnen werden. Alternativ kann eine Rektummanometrie erfolgen. Mit einem Kontrasteinlauf kann die Länge des aganglionären Segments bestimmt werden.

\section{Gedeihstörung, Malabsorption}

Eine Gedeihstörung ist eine Verzögerung der somatischen Entwicklung, bei der die Perzentilen von Gewicht, später auch der Größe unter die 3.Perzentile oder bezogen auf die genetische Zielgröße um mehr als 2 Hauptperzentilen abfällt. Ein Kreuzen der Perzentilen erfordert eine Diagnostik.

Bei einer Gedeihstörung sind folgende Ursachen zu bedenken:

- unzureichende Kalorienaufnahme: falsche Ernährung/Ernährungsgewohnheiten, Erbrechen/Dysphagie (z. B. gastroösophagealer Reflux, eosinophile Ösophagitis), chronisch konsumierende Erkrankungen (Organerkrankungen, chronische Inflammation, onkologische Erkrankungen)
- Maldigestion: Pankreasinsuffizienz (z. B. Mukoviszidose)

- Malabsorption: kongenitale Diarrhö, Dünndarmerkrankungen (Zöliakie, Nahrungsmittelallergie, CED, etc.), Cholestase

Zunächst ist die Gedeihstörung zu objektivieren. Dies erfolgt mittels Erfassung der anthropometrischen Daten wie Gewicht, Größe, Gewichts-, Wachstumsentwicklung und Body-Mass-Index mit Beurteilung der Perzentilenentwicklung und des Z-Scores (SDS).

Die Anamnese umfasst:

- Familienanamnese (Größe, Pubertätsentwicklung, Konsanguinität)

- Schwangerschaftsanamnese (Nikotin, Alkohol, Drogen)

- Geburtsanamnese (SGA, Asphyxie)

- Verlauf von Größen und Gewichtsentwicklung

- Meilensteine der Entwicklung

- psychosoziale Anamnese

- Ernährungsanamnese

- Stuhlanamnese

Bei Zweifeln an einer adäquaten Ernährung sollte ein Ernährungsprotokoll über 1 Woche protokolliert und ausgewertet werden.

Die klinische Untersuchung sollte neben den typischen Zeichen einer Gedeihstörung (Reduktion von Unterhautfettgewebe und Muskelmasse, ggf. Dehydratation) auch nach assoziierten Symptomen fahnden, die für bestimmte Grunderkrankungen typisch sind (Haut, Meteorismus, Stuhlbeschaffenheit, etc.).

\section{PRAXISTIPP}

Basislaboruntersuchungen bei Gedeihstörung BSG, BB, Elektrolyte, Ca, Ph, GOT, GPT, gGT, Glukose, Kreatinin, Albumin, Quick, aPPT, IGF1, IGFBP3, U-Status, GT-2, TSH

Zusätzlich sollten bei Hinweisen für eine Malabsorption weitere Laboruntersuchungen erfolgen.

Eine Abdomensonografie sollte immer durchgeführt werden. Die detaillierte Diagnostik bei spezifischen einzelnen Erkrankungsbildern ist in den entsprechenden Unterkapiteln dargestellt.

\section{Dysphagie (Schluckstörung)}

Störungen der Nahrungspassage durch den Ösophagus führen zur Dysphagie und zu Schluckproblemen. Gelegentlich werden Kinder primär notfallmäßig mit Nahrungsbolusingestion vorgestellt. Die zugrundeliegenden Erkrankungen können sein: 


\section{PRAXISTIPP}

Erweiterte Laboruntersuchungen bei Gedeihstörungen oder V.a. Malabsorption

Fe, Ferritin, Transferrin, Transferrinsättigung, IgA, IgG, IgE, Transglutaminase-AK, Folsäure, Vitamin B12, Vitamin D, Vitamin E, Zink, Cholesterin, Triglyceride, Gallensäuren, Blutgasanalyse Stuhl: Calprotectin, pH-Wert, reduzierende Substanzen, Pankreaselastase, Hämoccult, pathologe Keime/Parasiten (z. B. Giardia lamblia), ggf. Fettaussscheidung, ggf. Alpha-1-AntitrypsinAusscheidung im Stuhl, ggf. Schweißtest

- gastroösophagealer Reflux mit Ösophagitis

- Achalasie

- eosinophile Ösophagitis

Die Diagnostik der Refluxerkrankung wurde bereits im entsprechenden Kapitel dargestellt. Eine Achalasie ist mit einem Ösophagusbreischluck und/oder einer Ösphagusmanometrie zu erkennen. Eine eosinophile Ösophagitis kann nur endoskopisch/histologisch diagnostiziert werden. Bei jeder Bolusingestion bzw. Verdacht auf eosinophile Ösophagitis sollten daher Stufenbiopsien aus dem oberen Intestinaltrakt entnommen werden. Endoskopisch kann man charakteristische Schleimhautveränderungen sehen. Die Erkrankung ist wahrscheinlich, wenn mehr als 15 eosinophile Granulozyten pro HPF (high power field) in der Histologie gezählt werden. In ca. $60 \%$ liegt eine Nahrungsmittelallergie vor, die allerdings meist nicht IgE-vermittelt ist, so dass kein spezifisches lgE nachgewiesen werden kann und auch Prick-Tests keinen weiteren Aufschluss erlauben.

Merke

Bei Schluckstörungen sollte eine Ösophagoskopie durchgeführt werden.

\section{Bauchschmerzen}

Einer der häufigsten Vorstellungsgründe beim Kinderund Jugendarzt sind chronische bzw. rezidivierende Bauchschmerzen. Die möglichen Ursachen sind ausgesprochen vielfältig, so dass sich insbesondere bei diesen Beschwerden die Frage nach einem sinnvoll aufgebauten Diagnostikschema stellt, zumal die meisten Schmerzen funktioneller Natur sind. Eine immer weiterführende somatisch orientierte Diagnostik ist für den betroffenen Patienten und die Behandler frustrierend und führt eher zu einer Somatisierung und Schmerzverstärkung. Die Anamnese sollte gezielt nach Hinweisen für eine somatische Erkrankung fahnden, sehr sinnvoll ist die Verwendung eines standardisierten Bauchschmerzfragebogens, auf dem die sog.
Red Flags, die auf eine Organerkrankung hinweisen, dezidiert adressiert werden ( $\triangleright$ Abb. $\mathbf{7})$.

\section{PRAXISTIPP}

\section{Basisdiagnostik bei Bauchschmerzen}

BB, BSG, CRP, GOT, GPT, Bilirubin, LDH, Kreatinin, Harnstoff, IgA, IgG, IgE, Transglutaminase-IgA, Nahrungsmittelscreen Fx5 (Milcheiweiß, Hühnereiweiß, Kabeljau/Dorsch, Weizenmehl, Erdnuss, Sojabohne), Calprotectin im Stuhl, UrinStatus, abdominelle Sonografie

\section{Organische Erkrankungen}

Differenzialdiagnostisch müssen bei Bauchschmerzen folgende Erkrankungen bedacht werden:

- Gastrointestinaltrakt:

- gastroösophageale Refluxerkrankung

- Gastritis, Ulcus ventriculi, Ulcus duodeni (z. B. Helicobacter pylori)

- Zöliakie

- chronisch entzündliche Darmerkrankung

- chronische Cholecystitis

- chronische Hepatitis

- akute, chronische, rezidivierende Pankreatitis, pankreatische Pseudozyste

- granulomatöse Enterokolitis

- intestinale Tuberkulose

- Zuckerintoleranzen, -malabsoprtionen (Laktose, Fruktose, Zuckerersatzstoffe)

- parasitäre Erkrankungen (Giardia lamblia)

- postoperative Briden

- Nierensteine

- Ovarialzyste

- paroxysmale nächtliche Hämoglobinurie

- Pyelonephritis/Harnwegsinfekt

- systemische Erkrankungen:

- familiäres andioneurotisches Ödem

- familiäres Mittelmeerfieber

- Nahrungsmittelallergie

- Schwermetallvergiftung (Blei, Arsen)

- abdominelle Migräne

- abdominelle IgA-Vaskulitis (Purpura SchönleinHenoch)

- Porphyrie

- Sichelzellanämie

\section{Gastritis, Ulcus ventriuli, Ulcus duodeni}

Zur Diagnostik dieser Erkrankungen ist eine Ösophagogastroduodenoskopie notwendig. Ein invasiver (mittels Endoskopie) oder nicht invasiver (monoklonaler Stuhlantigentest mit ELISA) diagnostischer Test auf eine Helicobacter-pylori-Infektion (HP-Infektion) sollte bei Kindern und Jugendlichen nur durchgeführt werden, wenn im Falle eines positiven Testergebnisses eine Therapie vorgesehen ist. Bei einer Endoskopie 


\section{Bauchschmerzfragebogen}

$\begin{array}{lll}\text { Patient: } & \text { geb.: } & \text { Datum: } \\ \text { Ausgefüllt von: } \square \text { Patient } \quad \square \text { Mutter } \quad \square \text { Vater } \quad \text { anderer Person }\end{array}$

Seit wann bestehen die Bauchschmerzen regelmäßig?

\begin{tabular}{lll} 
seit ca. & \multicolumn{3}{l}{ Wochen/Monaten } & \\
Beginn nach einer Auslandsreise & $\square$ ja & $\square$ nein \\
nach einer akuten Durchfallerkrankung & $\square$ ja & $\square$ nein
\end{tabular}

Wie oft treten die Schmerzen auf? x pro Tag/Woche, $\square$ wechselnd

ggf. beschreiben

Wo ist der Schmerz lokalisiert? Andere Symptome?

$\square$ um den Bauchnabel $\square$ andere Lokalisation
liegt Sodbrennen vor?
liegen Schluckstörungen/Erbrechen vor? $\square$ ja $\square$ ja $\square$ nein
viel Luft im Bauch?

wo?
$\square$ gering (kaum Beeinträchtigung)
$\square$ deutlich (unterbricht Aktivität)
$\square$ stark (Schmerzäußerung, z.B. Weinen)
$\square$ wechselnd

Ist der Nachtschlaf durch Schmerzen unterbrochen?

$\square$ ja $\square$ nein

Ist der Schmerz tageszeitabhängig?

$\square$ ja $\square$ nein wann?

Treten die Schmerzen im Zusammenhang mit dem Stuhlgang auf?

$\square$ nein $\square$ vorher $\square$ nacher $\square$ beim Stuhlgang

Wie oft hast du/hat Ihr Kind Stuhlgang am Tag? x pro Tag/Woche

Wie ist die Stuhlkonsistenz? $\square$ normal $\square$ breiig/flüssig $\square$ hart

Besteht Stuhlschmieren (schmutzige Unterhose)? $\square$ ja $\square$ nein

Blutbeimengungen im Stuhl?

$\square$ ja $\square$ nein

Gibt es Störungen beim Wasserlassen?

$\square$ ja $\square$ nein

Stehen die Schmerzen in Zusammenhang mit bestimmten Nahrungsmitteln?

$\square$ Milch/Milchprodukte $\square$ Früchte/Fruchtsäfte $\square$ Fertiggetränke

andere

Was trinkst du/trinkt Ihr Kind als hauptsächlichen Durstlöscher?

Sind schon bestimmte Diätversuche durchgeführt worden?

welche?

wie lang?

mit Erfolg?

Abb.7 Bauchschmerzfragebogen. (Fortsetzung siehe folgende Seite) 


\section{Bauchschmerzfragebogen}

Sind medikamentöse Behandlungen erfolgt?

welche? wie lang?

mit Erfolg?

Welche Untersuchungen sind bislang durchgeführt worden?

Hast du/hat Ihr Kind an Gewicht abgenommen? $\quad \square$ ja $\quad \square$ nein

wie viel Kilogramm?

kg; in welcher Zeit? Wochen/Monate

Fehltage in Schule/Kindergarten? $\square$ nein/nicht nennenswert

wie viele Tage? in den letzten 3 Monaten/Halbjahr (ggf. Ca.-Angabe)

Treten die Schmerzen bei Stresssituationen vermehrt auf? $\quad \square$ ja $\quad \square$ nein

bei welchen Gelegenheiten?

Könnten psychologische Gründe eine Rolle spielen? $\square$ ja $\quad \square$ eher nein

Liegen andere Erkrankungen vor (z.B. Allergien, andere Schmerzlokalisationen, etc.)?

welche?

Sind in der Familie Magen-|Darmerkrankungen bekannt (z.B. Laktoseintoleranz, Allergien etc.)?

welche? bei wem?

Bist du/ist Ihr Kind abgesehen von den Bauchschmerzen gesund? $\quad \square$ ja $\quad \square$ nein

Möchten Sie noch etwas ergänzen?

Vielen Dank! Alles Weitere besprechen wir im persönlichen Gespräch.

sind Biopsien aus Antrum- und Korpusbereich durchzuführen. Zusätzlich wird ein Urease-Schnelltest bzw. eine HP-Kultur eines Biopsats empfohlen.

\section{Merke}

Bei Verdacht auf funktionelle Bauchschmerzen ist eine HP-Diagnostik nicht indiziert.

Bei Kindern mit erstgradigen Verwandten mit Magenkarzinom kann die endoskopische Diagnostik erwogen werden, ebenso bei Kindern mit Eisenmangelanämie nach Ausschluss anderer Ursachen. Der HP-Antigennachweis im Serum ist ohne Wert, eine eventuelle Eradikation sollte mit einem Stuhlantigentest oder einem C13-Atemtest überprüft werden [8].

\section{Funktionelle Bauchschmerzen}

Bezüglich der Diagnose bzw. Differenzialdiagnose funktioneller Bauchschmerzen sei auf die Rome-IVKriterien für das Kindes- und Jugendalter verwiesen [9]. Ein standardisierter Bauchschmerzfragebogen ( Abb.7) gibt wertvolle Hinweise bei funktionellen Beschwerden. Die Labordiagnostik sollte sich auf die Basisdiagnostik bei Bauchschmerzen beschränken.

Merke

Eine nicht indizierte endoskopische Diagnostik verstärkt bei funktionellen Bauchschmerzen die Somatisierungstendenz der Patienten bzw. Eltern und sollte daher vermieden werden. 
- Tab.4 Diagnostische und therapeutische Ösophagogastroduodenoskopie, Indikation, Nichtindikation und Kontraindikation.

\begin{tabular}{|l|l|}
\hline Indikationsart & Differenzialdiagnose \\
\hline $\begin{array}{l}\text { Diagnostische } \\
\text { Indikation }\end{array}$ & Gewichtsverlust, Gedeihstörung \\
\hline & unklare Anämie \\
\hline & Bauchschmerzen mit V.a. Organerkrankung \\
\hline & Schluckstörung \\
\hline Verätzung/V.a. Verätzung \\
\hline unklares rezidivierendes Erbrechen \\
\hline Hämatemesis \\
\hline unklarer chronischer Durchfall \\
\hline V.a. Graft-versus-Host-Erkrankung \\
\hline Chronische gastroösophageale Refluxerkrankung/ \\
\hline Barrett-Ösophagus \\
\hline perkutan endoskopische Gastrotomie (PEG)
\end{tabular}

\section{Gastrointestinale Blutung}

\section{Obere gastrointestinale Blutung}

Ursachen einer oberen gastrointestinalen (GI) Blutung sind:

- verschlucktes Blut aus dem Nasopharynxbereich

- Mallory-Weiss-Syndrom bei heftigem Erbrechen

- Ösophagitis

- Ösophagus-/Fundusvarizenblutung (portale Hypertension?)

- Ulcus/Gastritis

Die Anamnese sollte sorgfältig nach den genannten Krankheitsbildern fahnden, in der weiterführenden Diagnostik ist eine Ösophagogastroduodenoskopie mit ggf. Biopsiegewinnung notwendig.

\section{Hämatochezie, Meläna}

Blutauflagerungen auf dem Stuhl bei jungen Säuglingen (1 - 6 Wochen) sind das typische Zeichen einer allergischen Proktokolitis. Die Kinder sind sonst gesund, werden oft gestillt, seltener mit Formula-Ernährung ernährt. In der Regel ist keine weiterführende Diagnostik notwendig. Wenn man kolonoskopiert, findet sich eine follikuläre Hyperplasie der Dickdarmschleimhaut. Bei hartnäckigen Fällen sollte sich die Mutter kuhmilchfrei ernähren (ggf. auch eiweiß-, mais- und sojafrei). Bei Persistenz kann auch eine Therapienahrung (wie bei der Kuhmilchallergie) erwogen werden. Nach einigen Wochen verliert sich die Symptomatik spontan, es kommt zu einer Toleranz. Man geht davon aus, dass eine relevante Menge an Kuhmilchprotein über die Muttermilch an die Kinder übergeht.

\section{Merke}

Eine Hämatochezie im Säuglingsalter bei einem sonst gesunden Kind ist meist auf eine kuhmilchproteininduzierte Proktokolitis zurückzuführen. Eine Koloskopie ist nicht notwendig.

\section{Analfissur, Anitis}

Eine Hämatochezie verbunden mit schmerzhafter Defäkation und/oder Obstipationsneigung ist fast immer auf eine Analfissur zurückzuführen. Neben der Anamnese ist die klinische Untersuchung wichtig. Bei einem Analekzem kann eine Streptokokkenanitis vorliegen, die mittels Analabstrich diagnostiziert werden kann und systemisch mit Antibiotika behandelt werden muss.

\section{Juveniler Kolonpolyp}

Bei sonst gesunden Kleinkindern und jungen Schulkindern mit rezidivierenden Hämatochezieepisoden (gelegentlich auch mit Schleimbeimengungen) ist die wahrscheinlichste Verdachtsdiagnose ein oder mehrere juvenile Kolonpolypen. Zur Diagnostik ist eine Ileokoloskopie notwendig. Bei Nachweis eines Polypen kann dieser bei der Untersuchung mittels Diathermieschlinge abgetragen werden.

Andere bzw. seltenere Differenzialdiagnosen sind eine akute Enteritis (Stuhlkultur, z. B. Salmonellen), eine chronisch entzündliche Darmerkrankung, Gefäßdysplasien (Endoskopie, Histologie) oder ein Meckel-Divertikel (Meckelscan, Laparoskopie).

\section{Endoskopische Diagnostik}

In > Tab. 4 und > Tab. 5 sind die Indikationen zur diagnostischen und therapeutischen Endoskopien, die Nichtindikationen und die Kontraindikationen dargestellt [10]. 


\section{Sinnlose, entbehrliche Diagnostik}

\section{Mikroökologische Stuhldiagnostik}

Die Stuhldiagnostik beinhaltet teils sinnvolle Parameter (Calprotectin, Alpha-1-Antitrypsin-Ausscheidung), jedoch auch entbehrliche Diagnostik, wie die quantitative Keimanalyse. Es darf bezweifelt werden, dass die quantitative Keimanalyse mit der fast regelhaften Diagnose einer Dysbiose bei präanalytisch sehr unterschiedlich aufbewahrten Stuhlproben (Postversand, Lagerungsdauer, Temperaturbedingungen, etc.) sehr viel Sinn macht.

\section{Spezifische IgG-Diagnostik bei Nahrungsmittel- allergien}

Verzichtbar bzw. nicht indiziert sind auch folgende Diagnostiken:
- Bioresonanz
- Histaminfreisetzungstest
- Mediatorfreisetzungstest
- endoskopische Allergenprovokation

\section{Pankreaserkrankungen}

\section{Pankreatitis}

Bei Pankreaserkrankungen kann man primäre von sekundären Formen unterscheiden. Sie können akut, akut rezidivierend und chronisch auftreten. Klinische Symptom sind Oberbauchschmerzen, typischerweise gürtelförmig ausstrahlend, wobei die Schmerzcharakteristik im Kindesalter nicht immer klar erfassbar ist. In der Initialdiagnostik von Bauchschmerzen sollte eine Bestimmung der Lipase nicht fehlen. Sie ist der Amylase an Sensitivität und Spezifität überlegen. Die Höhe der Lipase muss nicht mit dem Schweregrad der Pankreatitis korrelieren. Bei Schüben der Erkrankung liegt eine akut rezidivierende bzw. chronische Pankreatitis vor. Langfristig kann eine chronische Pankreatitis mit einer erst exokrinen (Fettmalassimilation), später auch endokrinen Insuffizienz (Diabetes mellitus) des Organs einhergehen.

In der Anamnese muss nach familiären Pankreatitiden gefragt werden, nach Begleiterkrankungen des Patienten mit entsprechender Medikation, bei der Untersuchung sollten Hinweise für Infektionen berücksichtigt werden (Viren, Bakterien, Parasiten).

\section{PRAXISTIPP}

\section{Basislabor bei Pankreatitis}

Lipase, Blutbild, Blutgasanalyse, Blutsenkungsgeschwindigkeit, Elektrolyte, Triglyceride, Kreatinin, Harnstoff, GPT, LDH, CPR, Glukose
- Tab. 5 Diagnostische und therapeutische lleokoloskopie, Indikation, Nichtindikation und Kontraindikation.

\begin{tabular}{|c|c|}
\hline Art & Indikation \\
\hline \multirow{9}{*}{$\begin{array}{l}\text { diagnostische } \\
\text { Indikation }\end{array}$} & unklare Anämie \\
\hline & unklarer chronischer Durchfall \\
\hline & perianale Läsionen (Fisteln, Abszesse) \\
\hline & rektale Blutung \\
\hline & unklare Gedeihstörung \\
\hline & V.a. Graft-versus-Host-Erkrankung \\
\hline & $\begin{array}{l}\text { Abstoßung oder Komplikation nach Darm- } \\
\text { transplantation }\end{array}$ \\
\hline & radiologische Darstellung einer Stenose/Striktur \\
\hline & Polyposis-Syndrome/Dickdarmpolypen \\
\hline \multirow{5}{*}{$\begin{array}{l}\text { therapeutische } \\
\text { Indikation }\end{array}$} & Polypektomie \\
\hline & Dilatation von Stenosen \\
\hline & Behandlung von Blutungen \\
\hline & Fremdkörperentfernung \\
\hline & Sigmavolvulus \\
\hline \multirow[t]{2}{*}{ Nichtindikationen } & funktionelle gastrointestinale Beschwerden \\
\hline & Verstopfung \\
\hline \multirow[t]{2}{*}{ Kontraindikationen } & Kolonperforation \\
\hline & Z.n. intestinaler Resektion (<7 Tage) \\
\hline
\end{tabular}

Die weitere Diagnostik (Labor, Molekulargenetik, Bildgebung) richtet sich nach Hinweisen auf die grundsätzlich differenzialdiagnostisch zu erwägenden Erkrankungen ( Tab.6) [11].

Die exokrine Insuffizienz des Pankreas erfasst man mit der Bestimmung der Pankreaselastase und/oder der Stuhlfettbestimmung. Alle anderen Verfahren (z. B. Sekretin-Pankreozymin-Test) werden heute wegen fehlender Sensitivität bzw. Spezifität oder Unpraktikabilität kaum noch angewendet.

Die endokrine Insuffizienz mit einer prädiabetischen bzw. diabetischen Stoffwechsellage lässt sich diagnostizieren mit:

- Glukoseausscheidung (U-Stix)

- Glukosetagesprofil

- oralem Glukosetoleranztest

- Bestimmung des HBA1c (Langzeitparameter)

Eine akute Pankreatitis ist meist inflammatorischödematös und mittelfristig selbstlimitierend. Es kann sich aber eine schwere nekrotisierende Pankreatitis entwickeln. Ein CRP> $12 \mathrm{mg} / \mathrm{dl}$ spricht für einen nekrotisierenden Verlauf. Dabei entstehende Abszedierun- 
Tab.6 Differenzialdiagnose einer chronischen Pankreatitis [11].

\begin{tabular}{|c|c|}
\hline Erkrankung & Differenzialdiagnose \\
\hline $\begin{array}{l}\text { hereditär/idiopathisch } \\
\text { (Molekulardiagnostik) }\end{array}$ & $\begin{array}{l}\text { - } \text { kationisches Trypsinogen (PRSS1) } \\
\text { - Caboxipeptidase A1 (CPA1) } \\
\text { - Serinproteaseinhibitor, Kazal Typ-1 (SPINK1) } \\
\text { - Chymotrypsin C (CTRC) } \\
\text { - Cystic Fibrosis Transmembrane Conductance } \\
\text { Regulator (CFTR) } \\
\text { " ggf. andere (Cathepsin B, Calcium-sensing } \\
\text { Receptor Gene, Claudin-2-locus X-Chromo- } \\
\text { som) }\end{array}$ \\
\hline $\begin{array}{l}\text { systemische } \\
\text { Erkrankungen }\end{array}$ & $\begin{array}{l}\text { - Kollagenosen (chronisch entzündliche } \\
\text { Darmerkrankungen, primär sklerosierende } \\
\text { Cholangitis, M. Beçet, Lupus erythematodes, } \\
\text { rheumatoide Arthritis, Panarteritis nodosa) } \\
\text { " zystische Fibrose (Schweißtest, Genetik) } \\
\text { " autoimmune Pankreatitis (IgG 4) }\end{array}$ \\
\hline $\begin{array}{l}\text { metabolische } \\
\text { Erkrankungen }\end{array}$ & $\begin{array}{l}\text { - Hypertriglyceridämie } \\
\text { - Hyperkalzämie } \\
\text { - Dystrophie, Malnutrition } \\
\text { " diabetische Ketoazidose } \\
\text { - Niereninsuffizienz }\end{array}$ \\
\hline Medikamente/Toxine & $\begin{array}{l}\text { - Asparaginase } \\
\text { - Azathioprin } \\
\text { - Didanosin } \\
\text { - Kalzium } \\
\text { - 6-Mercaptopurin } \\
\text { - Östrogene } \\
\text { - Statine } \\
\text { - Stibogluconat } \\
\text { - Suldinac } \\
\text { - Valproat } \\
\text { - Vincristin }\end{array}$ \\
\hline $\begin{array}{l}\text { mechanisch/ } \\
\text { strukturelle Ursachen } \\
\text { (Bildgebung) }\end{array}$ & $\begin{array}{l}\text { - anatomische Anomalien (Choledochuszyste, } \\
\text { Pancreas anulare, Pankreasgangduplikaturen, } \\
\text { evtl. Pancreas divisum) } \\
\text { " Obstruktion (Gallensteine, Tumoren, Para- } \\
\text { siten) } \\
\text { " Trauma }\end{array}$ \\
\hline Infektionen & $\begin{array}{l}\text { - Viren (Enteroviren, Herpesviren, Hepatitis } \\
\text { A, B, E, HIV, Masern, Mumps, Röteln) } \\
\text { - Bakterien (E. coli, Campylobakter, Legionellen, } \\
\text { Leptospiren, Mykoplasmen, Salmonellen, } \\
\text { Yersinien) } \\
\text { - Parasiten (Ascaris lumbricoides, Clonorchis } \\
\text { sinensis, Cryptosporidium parvum, Echino- } \\
\text { kokken, Fasciola hepatica, Toxoplasma } \\
\text { gondii) }\end{array}$ \\
\hline
\end{tabular}

gen lassen sich am besten durch die kontrastmittelverstärkte Computertomografie erkennen.

\section{Exokrine Pankreasinsuffizienz}

Eine exokrine Pankreasinsuffizienz kann sich in der Folge einer chronischen Pankreatitis entwickeln, es gibt aber auch primäre Ursachen. Dazu zählen:

- isolierte Enzymdefekte (Lipase, Kolipase, Trypsinogen und Alpha-Amylase)

- Shwachman-Dimond-Syndrom (metaphysäre Dysplasie, Rippenverkürzung, Klinodaktylie, zyklische

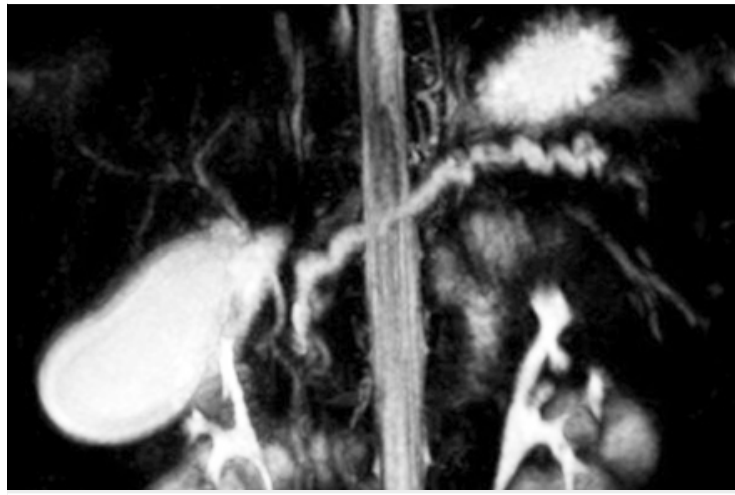

Abb. 8 MRCP: erweiterter Ductus pancreaticus bei chronischer Pankreatitis.

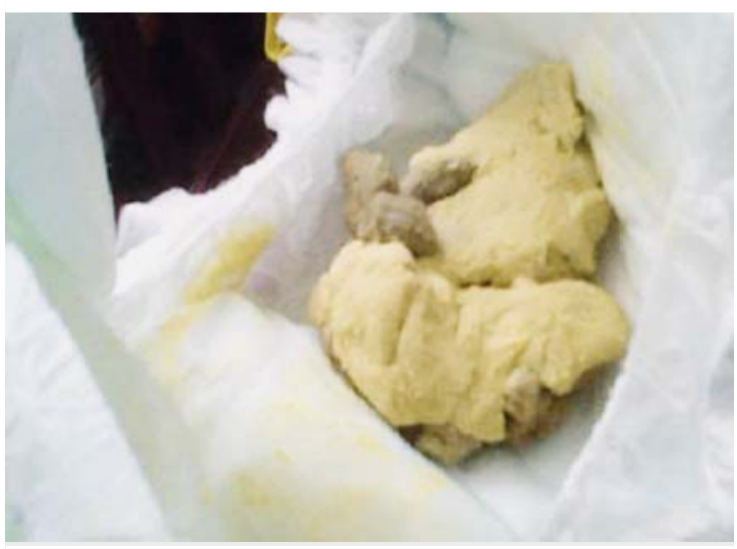

Abb. 9 Acholischer Stuhl.

Neutropenie, mentale Retardierung, Infektanfälligkeit)

- Pearson-Syndrom (multisystemische mitochondriale Zytopathie)

- Johanson-Blizzard-Syndrom

Bezüglich der Einzelheiten sei auf die weiterführende Literatur verwiesen.

\section{Bildgebung}

Die bildgebende Diagnostik umfasst die Sonografie, Endosonografie, die kontrastmittelverstärkte CT, die sekretinstimulierte MRCP (Magnetresonanzcholangiopankreatikografie, - Abb.8) und die ERCP (endoskopisch retrograde Cholangiopankreatikografie).

\section{Leber- und Gallenwegs- erkrankungen}

\section{Neonatale Cholestase}

Ein Neugeborenikterus ist häufig und erfordert in der Regel keine spezifische Diagnostik oder therapeutische Maßnahmen, wenn das Bilirubin unterhalb der Photo- 


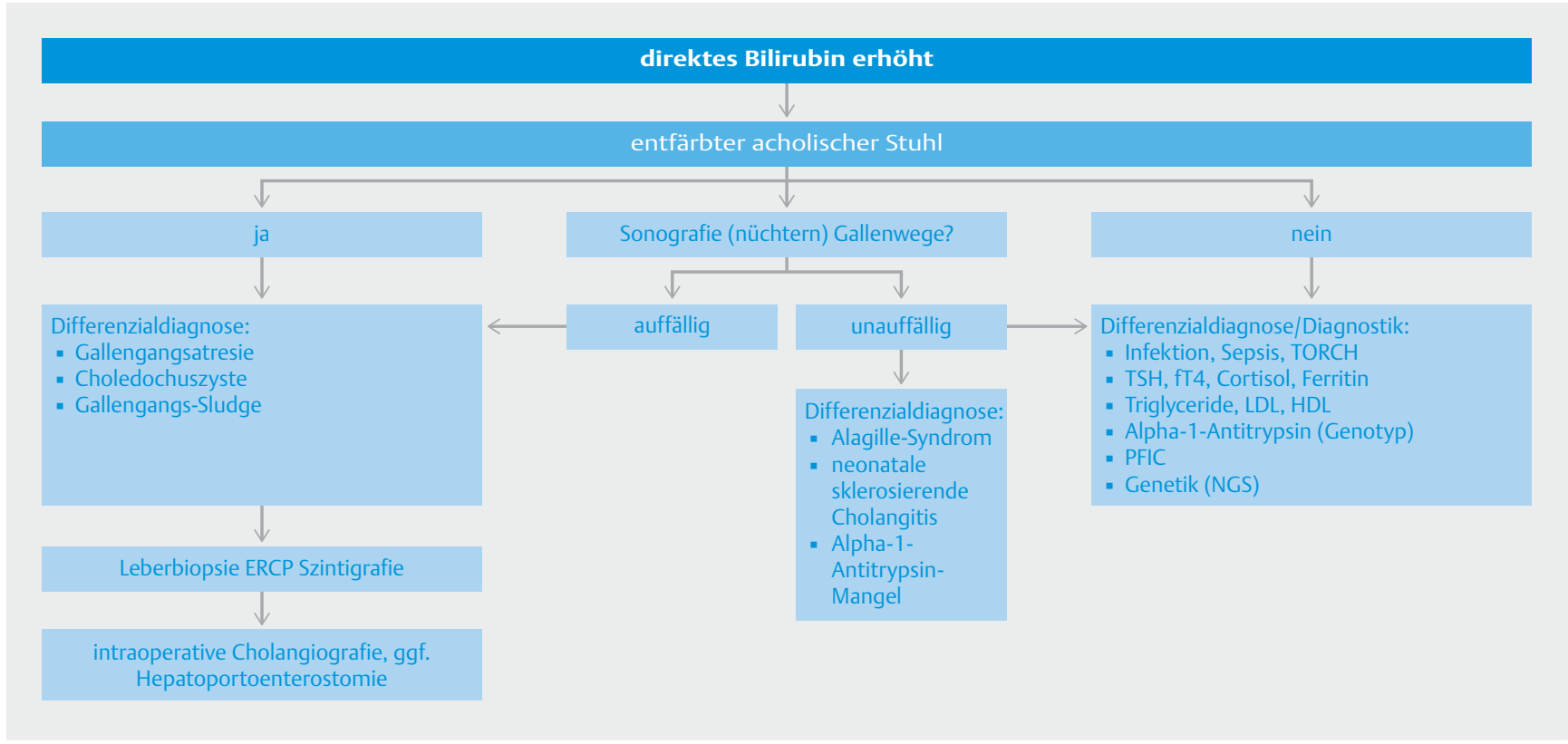

Abb.10 Diagnostischer Algorithmus zur neonatalen Cholestase [15].

therapiegrenzen bleibt [12]. Bei einem Ikterus gravis (Bilirubin >14 g/dl bzw. $238 \mu \mathrm{mol} / \mathrm{l}$ ) oder einem Ikterus prolongatus (>14 Tage bzw.>21 Tage bei gestillten Kindern) sollte neben einem Morbus hämolyticus neonatorum auch an eine cholestatische Lebererkrankung gedacht werden und eine Differenzierung des Bilirubins vorgenommen werden (indirektes vs. direktes Bilirubin). Dies gilt insbesondere, wenn ein acholischer Stuhl vorliegt (> Abb.9). Der Entschluss des Gemeinsamen Bundesausschusses zur Integration der Stuhlfarbenkontrolle in die kinderärztlichen Vorsorgeuntersuchungen (U3) ist ein wichtiger Schritt zur weiteren Sensibilisierung von niedergelassenen kinderärztlichen Kollegen. Unter dem folgenden Link ist eine Stuhlkarte für Eltern zu finden: https://www.aekn.de/ fileadmin/media/Downloadcenter/Fortbildung/StuhlKarte_mhh_26_7_16.pdf (Zugriff am 22.2.2017).

Eine neonatale Cholestase hat eine Prävalenz von ca. 1:2500 [13]. Obwohl die Zahl der unterschiedlichen Krankheitsursachen sehr hoch ist, liegen etwa 95\% aller neonatalen Cholestasen nur 10 unterschiedliche Krankheiten zugrunde [14].

\section{Merke}

Bei der neonatalen Cholestase ist eine rasche Diagnostik erforderlich, da es Erkrankungen gibt, die nur bei schneller Einleitung einer spezifischen Therapie effektiv zu behandeln sind.

In $>$ Abb. 10 ist der diagnostische Algorithmus zur neonatalen Cholestase dargestellt.
Merke

Ein neonataler Ikterus kann ein frühes Zeichen einer Lebererkrankung sein. Bei Bilirubin $>14 \mathrm{~g} / \mathrm{dl}$ und/oder über den 14. Lebenstag (21. Tag bei gestillten Kindern) hinaus muss das direkte Bilirubin bestimmt werden.

\section{Labordiagnostik}

Die Labordiagnostik umfasst im 1. Schritt die in der Box genannten Parameter.

\section{PRAXISTIPP}

\section{Basislabor bei neonataler Cholestase}

Bestimmung des Ausmaßes der Cholestase (direktes Bilirubin, yGT, Gallensäuren), des Leberzellschadens (ALAT, ASAT, GLDH), der Leberfunktionsbeeinträchtigung (CHE, Albumin, Gerinnungsanalytik), sowie zusätzliche Parameter (Hämolyseparameter, Lipase, Glukose, Laktat, Ammoniak, CRP).

Im 2. Schritt folgt die Klärung der Genese der Cholestase. Entfärbte acholische Stühle sollten eine unverzügliche weiterführende Diagnostik nach sich ziehen. In - Tab. 7 sind die verschiedenen Ursachen einer neonatalen Cholestase mit den wichtigsten diagnostischen Markern dargestellt.

\footnotetext{
Merke

Bei acholischen, entfärbten Stühlen im Neugeborenen- und jungen Säuglingsalter ist Eile geboten.
} 
Tab.7 Ursachen der neonatalen Cholestase sowie deren zielführende Diagnostik in Klammern.

\begin{tabular}{|c|c|}
\hline Diagnostik & Ursache \\
\hline $\begin{array}{l}\text { Infektionen } \\
\text { (Serologie, Infektionsparameter) }\end{array}$ & $\begin{array}{l}\text { " bakterielle Infektion (Sepsis, Harnwegsinfektionen) } \\
\text { " TORCH (Toxoplasmose, Röteln, CMV, Herpes) } \\
\text { " Epstein-Barr-Virus, Enteroviren, Adenoviren, HIV, Hepatitis B }\end{array}$ \\
\hline $\begin{array}{l}\text { Gallenwegserkrankungen } \\
\text { (Bildgebung: Sonografie, ERC, Histologie, } \\
\text { Szintigrafie) }\end{array}$ & $\begin{array}{l}\text { " Gallengangsatresie } \\
\text { " Choledochuszyste } \\
\text { " Caroli-Syndrom } \\
\text { " Syndrom der eingedickten Galle } \\
\text { " Gallensteine } \\
\text { " spontane Gallenwegsperforation } \\
\text { " neonatale sklerosierende Cholangitis }\end{array}$ \\
\hline $\begin{array}{l}\text { Stoffwechselerkrankungen, genetische } \\
\text { Erkrankungen } \\
\text { (Stoffwechseldiagnostik, Genetik) }\end{array}$ & $\begin{array}{l}\text { " Alpha-1-Antitrypsinmangel (quantitative Bestimmung, Genotypisierung) } \\
\text { " Alagille-Syndrom (syndromaler Habitus, Genetik) } \\
\text { " progressive familiäre intrahepatische Cholestase (yGT erniedrigt, Lipidelektrophorese, } \\
\text { Genetik) } \\
\text { " Galaktosämie (Stoffwechselscreening, reduzierende Substanzen im Urin) } \\
\text { " Tyrosinämie Typ I (Aminosäuren im Plasma, Succinylaceton im Urin) } \\
\text { - Fettstoffwechselerkrankungen: } \\
\text { - M. Nieman-Pick Typ C (Speicherzellen im Knochenmark) } \\
\text { - M. Gaucher (ß-Cerebrosidase in Leukozyten oder Fibroblasten) } \\
\text { - M. Wolman (Cholesterin, Triglyceride) } \\
\text { - Mitochondriopathien (Zellweger-Syndrom, Atmungskettendefekte) (VLDFA, Blutgasanalyse, } \\
\text { - Laktat) } \\
\text { " zystische Fibrose (Schweißtest, Genetik) } \\
\text { " Gallensäurestoffwechselstörungen (Synthesedefekte, Dubin-Johnson-Syndrom, Rotor-Syn- } \\
\text { drom) (Gallensäureanalytik, FAB-Massenspektrometrie im Urin, Genetik) } \\
\text { - Aagenaes Syndrom } \\
\text { - Arginasemangel (Aminosäuren Plasma/Urin) }\end{array}$ \\
\hline $\begin{array}{l}\text { endokrinologische Erkrankungen } \\
\text { (endokrinologische Diagnostik) }\end{array}$ & $\begin{array}{l}\text { " Hypopituitarismus } \\
\text { " Hypothyreose } \\
\text { " Hypoadrenalismus (Nebenniereninsuffizienz) }\end{array}$ \\
\hline $\begin{array}{l}\text { chromosomale Erkrankungen } \\
\text { (Chromosomenanalyse) }\end{array}$ & $\begin{array}{l}\text { - Trisomie 21, 13, } 18 \\
\text { " Turner-Syndrom }\end{array}$ \\
\hline $\begin{array}{l}\text { Alloimmunerkrankungen (neonatale } \\
\text { Hämochromatose, Ferritin }>1000 \mu \mathrm{g} / \mathrm{l} \text {, Siderose } \\
\text { in der Leber, Speicheldrüsen [Biopsie], MRT, } \\
\text { Fe erhöht) }\end{array}$ & diplazentar übertragene Antikörper gegen fetale Hepatozyten \\
\hline $\begin{array}{l}\text { vaskuläre Erkrankungen } \\
\text { (Sonografie, anderes) }\end{array}$ & $\begin{array}{l}\text { " Budd-Chiari-Syndrom } \\
\text { " perinatale Asphyxie } \\
\text { " Hämangioendotheliome } \\
\text { " Herzversagen }\end{array}$ \\
\hline toxische Erkrankungen (Anamnese) & $\begin{array}{l}\text { " parenterale Ernährung } \\
\text { - fetales Alkoholsyndrom }\end{array}$ \\
\hline Verschiedenes & $\begin{array}{l}\text { - familiäre hämophagozytotische Lymphohistiozytose } \\
\text { - ARC-Syndrom (Arthrogryposis, renal tubuläre Erkrankung, Cholestase) }\end{array}$ \\
\hline
\end{tabular}

In der Diagnostik kommt der abdominellen Sonografie eine entscheidende Bedeutung zu. Die Gallengangsatresie zählt immerhin zu den häufigsten Ursachen einer neonatalen Cholestase ( $>$ Tab. 8) [14]. Nur bei einer operativen Wiederherstellung des Gallenflusses in Form einer Hepatoportoenterostomie innerhalb dieses Zeitfensters kann das sonst unausweichliche Schicksal der Entwicklung einer Leberzirrhose mit terminalem Organversagen vermieden werden.
Merke

Die Diagnose einer Gallengangsatresie darf nicht verzögert werden, da die chirurgische initiale Therapie einer Hepatoportoenterostomie nach Kasai bis spätestens zum 60. Lebenstag erfolgen muss. 


\section{Bildgebung}

Die bildgebende Diagnostik ist insbesondere bei der Gallengangsatresie wichtig. Die Sonografie bietet erste Hinweise mit der Darstellung einer rudimentären oder gar fehlenden Gallenblase, beeinträchtigter Kinetik der Gallenblase (keine postprandiale Entleerung), Darstellung eines echoreichen Bandes über der Pfortaderbifurkation (triangular cord sign), subkapsuläre arterielle Hypervaskluarisation der Leber, ist aber nicht ausreichend spezifisch.

Eine hepatobiliäre Szintigrafie hat eine hohe Sensitivität $(98,7 \%)$ bei jedoch auch nur geringer Spezifität $(70,4 \%)$ [16]. Sie ist zeitaufwändig und verzögert manchmal die Diagnostik, so dass sie seltener eingesetzt wird.

An erfahrenen Zentren gewinnt die endoskopisch retrograde Cholangiografie (ERC) zunehmend an Bedeutung. Die spätestens intraoperative Cholangiografie bei der operativen Exploration bestätigt die Diagnose. Die Leberbiopsie hat mit einer Spezifität von über $90 \%$ weiterhin ihren festen Platz in der Differenzialdiagnostik.

Eine gezielte Überprüfung der häufigsten Ursachen einer neonatalen Cholestase ( $\triangleright$ Tab.8) ist der 1.Schritt im Sinne der rationalen Diagnostik. Dazu gehört:

- Sonografie (obstruktive Gallenwegserkrankungen, Gallengangsatresie)

- quantitative Alpha-1-Antitrypsin-Bestimmung, ggf. Genotypisierung bei niedrigen oder normalen Werten

- syndromaler Habitus, yGT, Lipidelektrophorese, ggf. Genetik (Alagille-Syndrom, progressive familiäre intrahepatische Cholestase - PFIC)

- Infektionsparameter, Virusserologie

- TSH (Hypothyreose, Hypopituitarismus)

- Neugeborenen-Screening, reduzierende Substanzen im Urin

(Galaktosämie)

Erst im weiteren Verlauf sollte bei nicht zur Diagnose führenden Befunden die Diagnostik erweitert werden ( $>$ Tab.6). Eine Vielzahl der hereditären metabolischen Erkrankungen lassen sich mittlerweile mit einer NextGeneration-Sequencing-Methode (NGS) diagnostizieren. Dabei wird DNA aus einer EDTA-Probe $(1-2 \mathrm{ml})$ extrahiert und anschließend Millionen DNA-Fragmente in einem einzigen Sequenzierlauf parallel sequenziert. Bis heute sind mehr als 120 Gene bekannt, die bei Hepatopathien eine Rolle spielen.

Merke

Zukünftig wird die Differenzialdiagnostik der neonatalen Cholestase mit den genetischen Untersuchungsmöglichkeiten sehr vereinfacht [17].
> Tab. 8 Häufigkeit der Ursachen einer neonatalen Cholestase [14].

\begin{tabular}{|l|l|}
\hline Diagnose & Anteil in \% \\
\hline idiopathische neonatale Hepatitis & 15 \\
\hline extrahepatische Gallengangsatresie & $25-30$ \\
\hline Alpha-1-Antitrypsin-Mangel & $7-10$ \\
\hline familiäre Cholestase-Syndrome (Alagille, PFIC) & $22-25$ \\
\hline bakterielle Sepsis & 2 \\
\hline CMV-Infektion & $3-5$ \\
\hline andere Virusinfektionen & 1 \\
\hline Endokrinopathien (Hypothyreose, Hypopituitarismus) & 1 \\
\hline Galaktosämie & 1 \\
\hline
\end{tabular}

\section{Transaminasenerhöhung jenseits der Neugeborenenperiode}

Grundsätzlich sind viele der genannten Erkrankungen auch in der Differenzialdiagnostik von Hepatopathien jenseits des Neugeborenenalters zu bedenken. In - Tab. 9 sind die unterschiedlichen möglichen Ursachen dargestellt.

Zunächst erfolgt die Basisdiagnostik bzw. das Basislabor. Im Sinne eines rationalen Vorgehens sollte die ätiologische Primärdiagnostik zunächst auf die wichtigsten Erkrankungen fokussiert werden. Dazu gehören:

- Sonografie (obstruktive Gallenwegserkrankungen, fokale Lebererkrankungen, Hinweise auf chronische Hepatopathie, nicht alkoholische Fettlebererkrankung-NAFLD)

- Infektionsparameter, Virusserologien

- Autoimmunparameter ANA, SLA, LKM1, LKM3, SMA, LC1, ANCA (Autoimmunhepatitis, autoimmun sklerosierende Cholangits, primär sklerosierende Cholangitis, „Overlap“-Syndrom, De-novo-Autoimmunhepatitis nach Lebertransplantation)

- Kupfer, Coeruloplasmin, Kupferausscheidung im Urin (M. Wilson)

Im weiteren Verlauf sollte bei nicht zur Diagnose führenden Befunden die Diagnostik erweitert werden. Findet sich weder in der Anamnese noch der klinischen Untersuchung bzw. laborgestützten Diagnostik eine klare Ursache, ist eine Leberbiopsie angezeigt. Dabei sollte neben einer Standardhistologie auch eine Bestimmung des Kupfergehalts durchgeführt werden, um einen M. Wilson auszuschließen (Kupfer $>250 \mathrm{mg} / \mathrm{g}$ Trockengewicht). 
$\checkmark$ Tab.9 Ursachen einer Hepatopathie im Kleinkind-, Schulkind- und Jugendalter.

\begin{tabular}{|c|c|}
\hline Art & Ursache \\
\hline infektiöse Hepatitis & $\begin{array}{l}\text { - Virusinfektionen: } \\
\text { - Hepatitis A, B, C, D, E } \\
\text { - hepatotrophe Viren (EBV, CMV, HSV, VZ, Adenoviren, Enteroviren, Röteln, etc.) } \\
\text { " bakterielle, parasitäre Infektionen: } \\
\text { - Bruzellose, Leptospirose, Echinokokkose, Toxoplasmose } \\
\text { - Q-Fieber, Amöbiasis, Schistosomiasis }\end{array}$ \\
\hline Gallenwegserkrankungen & - Choledocholithiasis \\
\hline Autoimmunerkrankungen der Leber & $\begin{array}{l}\text { - Autoimmunhepatitis (AIH) } \\
\text { " primär sklerosierende Cholangitis (PSC) } \\
\text { " primär biliäre Zirrhose (PBC) }\end{array}$ \\
\hline nicht alkoholische Steatosis hepatis (NASH) & - Ernährung, Adipositas \\
\hline Stoffwechselerkrankungen & $\begin{array}{l}\text { - s. Ursachen der neonatalen Cholestase ( }>\text { Tab. } 6 \text { ) } \\
\text { - Morbus Wilson } \\
\text { - hereditäre Hämochromatose } \\
\text { - Porphyrien (akute hepatische Porphyrie, chronische hepatische Porphyrie, erythropoeti- } \\
\text { sche Porphyrie) }\end{array}$ \\
\hline Medikamente & $\begin{array}{l}\text { - Anästhetika } \\
\text { - Hypnotika, Sedativa, Anxiolytika } \\
\text { - Neuroleptika, Antidepressiva } \\
\text { - Antiepileptika } \\
\text { - Analgetika, nicht steroidale Antiphlogistika } \\
\text { " Antibiotika } \\
\text { - Tuberkulostatika } \\
\text { - Antimykotika }\end{array}$ \\
\hline Toxine & $\begin{array}{l}\text { - German/Indian Childhood Disease (Kupferintoxikation) } \\
\text { " Knollenblätterpilz } \\
\text { " Organophosphate } \\
\text { - Alkohol }\end{array}$ \\
\hline Perfusionsschäden & $\begin{array}{l}\text { - Budd-Chiari-Syndrom } \\
\text { " Veno-occlusive Disease } \\
\text { " Hypotension/Hypoxie/Schock }\end{array}$ \\
\hline Begleithepatopathie bei Systemerkrankungen & $\begin{array}{l}\text { - Sepsis } \\
\text { - Diabetes mellitus } \\
\text { " Kawasaki-Syndrom } \\
\text { " chronisch entzündliche Darmerkrankungen }\end{array}$ \\
\hline Lebertumore & $\begin{array}{l}\text { " benigne Raumforderungen } \\
\text { " hepatozelluläre Karzinome } \\
\text { - maligne mesenchymale Tumoren }\end{array}$ \\
\hline Leberzirrhose unterschiedlicher Genese & - Verschiedenes \\
\hline akutes Leberversagen unterschiedlicher Genese & - Verschiedenes \\
\hline
\end{tabular}


KERNAUSSAGEN

- Die Kindergastroenterologie lässt sich in verschiedene Organerkrankungen unterteilen: in Erkrankungen des Intestinaltrakts, des Pankreas, der Leber und der ableitenden Gallenwege.

- Die diagnostischen Möglichkeiten der verschiedenen Erkrankungen sind vielfältig und erstrecken sich von der Molekulardiagnostik bis zur Endoskopie.

- Eine unkritische Anwendung oder „Auftragserteilung " an den Kindergastroenterologen sollte vermieden werden.

- Sehr viel sinnvoller ist eine gestufte Diagnostik unter Kenntnis der wichtigen anamnestischen Daten, der klinischen Befunde und der möglichen und wahrscheinlichen Differenzialdiagnosen.

- Es ist wichtig, die betroffenen Kinder und Jugendlichen nicht einer unnötigen Diagnostik zu unterwerfen, auch um bei den sehr häufigen funktionellen Störungen keiner weiteren Somatisierungstendenz Vorschub zu leisten.

\section{Interessenkonflikt}

Der Autor gibt an, dass kein Interessenkonflikt vorliegt.

\section{Über die Autoren}

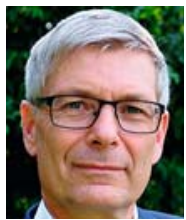

\section{Burkhard Rodeck}

Jahrgang 1954, Priv.-Doz. Dr. med., Promotion Heinrich-Heine Universität Düsseldorf. 1981-1982 Assistenzarzt Kinderchirurgische Klinik Klinikum Karlsruhe. 1982-1987 Facharztweiterbildung Kinder- und Jugendmedizin Medizinische Hochschule Hannover, Zusatzweiterbildung Kindergastroenterologie, Schwerpunkt Neonatologie. 1991-2000 Oberarzt am Zentrum für Kinder- und Jugendmedizin, Sektionsleiter Kindergastroenterologie und pädiatrische Lebertransplantationsmedizin, Habilitation an der Medizinischen Hochschule Hannover. 2001-2011 Chefarzt der Klinik für Kinderund Jugendmedizin am Marienhospital Osnabrück. Seit 2011 Chefarzt am Christlichen Kinderhospital Osnabrück.

\section{Korrespondenzadresse}

PD Dr. med. Burkhard Rodeck

Christliches Kinderhospital Osnabrück

Kinder-Gastroenterologie

Johannisfreiheit 1

49074 Osnabrück

E-Mail: B.Rodeck@ckos.de
[1] Beninga MA, Nurko S, Faure $C$ et al. Childhood functional gastrointestinal disorders: neonate/toddler. Gastroenterology 2016; 150: $1443-1455$

[2] Koletzko S, Niggemann B, Arato A et al. European Society of Pediatric Gastroenterology, Hepatology, and Nutrition. Diagnostic approach and management of cow's-milk protein allergy in infants and children: ESPGHAN GI Committee practical guidelines. J Pediatr Gastroenterol Nutr 2012; 55 : $221-229$

[3] Vandenplas Y, Dupont C, Eigenmann P et al. A workshop report on the development of the Cow's Milk-related Symptom Score awareness tool for young children. Acta Paediatr 2015; 104: 334-339

[4] Harb T, Matsuyama M, David M et al. Infant Colic-What works: A Systematic Review of Interventions for Breast-fed Infants. J Pediatr Gastroenterol Nutr 2016; 62: 668-686

[5] Posovsky C. Congenital intestinal diarrhoeal diseases: A diagnostic and therapeutic challenge. Best Pract Res Cin Gastroentrol 2016; 30: 187-211

[6] Levine A, Koletzko S, Turner D et al. European Society of Pediatric Gastroenterology, Hepatology, and Nutrition. ESPGHAN revised porto criteria for the diagnosis of inflammatory bowel disease in children and adolescents. J Pediatr Gastroenterol Nutr 2014; 5: 795-806

[7] Husby S, Koletzko S, Korponay-Szabó IR et al. ESPGHAN Working Group on Coeliac Disease Diagnosis; ESPGHAN Gastroenterology Committee; European Society for Pediatric Gastroenterology, Hepatology, and Nutrition. European Society for Pediatric Gastroenterology, Hepatology, and Nutrition guidelines for the diagnosis of coeliac disease. J Pediatr Gastroenterol Nutr 2012; 54: 136-160

[8] Fischbach W, Malfertheiner P, Lynen Jansen P et al. S2k-guideline Helicobacter pylori and gastroduodenal ulcer disease. Z Gastroenterol 2016; 54: 327-363

[9] Hyams JS, Di Lorenzo C, Saps M et al. Functional Disorders: Children and Adolescents. Gastroenterology 2016; 150: $1456-1468$

[10] Tringali A, Thomson M, Dumonceau JM et al. Pediatric gastrointestinal endoscopy: European Society of Gastrointestinal Endoscopy (ESGE) and European Society for Paediatric Gastroenterology Hepatology and Nutrition (ESPGHAN) Guideline Executive summary. Endoscopy 2017; 49: 83 - 91

[11] Witt H. Pankreatitis. In: Rodeck B, Zimmer KP, Hrsg. Pädiatrische Gastroenterologie, Hepatologie und Ernährung. 2. Aufl Heidelberg: Springer; 2013: $557-570$

[12] Rodeck B. Neonatale Cholestase. Päd up2date 2008; 3: $125-144$

[13] Fawaz R, Baumann U, Ekong U et al. Guideline for the Evaluation of Cholestatic Jaundice in Infants: Joint Recommendations of the North American Society for Pediatric Gastroenterology, Hepatology, and Nutrition (NASPGHAN) and the European Society for Pediatric Gastroenterology, Hepatology, and Nutrition (ESPGHAN). J Pediatr Gastroenterol Nutr 2017; 64: $154-168$

[14] McLin V, Balistreri W. Approach to Neonatal Cholestasis. In: Durie PR, Hamilton JR, Walker-Smith J, Watkins JB , eds. Pediatric Gastrointestinal Disease: Pathophysiology, Diagnosis, Management. Philadelphia: B.C. Decker; 2004: 10791093 
[15] Götze T, Blessing H, Grillhösl C et al. Neonatal Cholestasis Differential Diagnoses, Current Diagnostic Procedures, and Treatment. Front Pediatr 2015; 43: DOI: 10.3389/ fped.2015.00043

[16] Kianifar HR, Tehranian S, Shojaei P et al. Accuracy of hepatobiliary scintigraphy for differentiation of neonatal hepatitis from biliary atresia: systematic review and meta-analysis of the literature. Pediatr Radiol 2013; 43: 905-919

[17] Sturm E, Hörtnagel K. Genetische Diagnostik bei Lebererkrankungen im Kindesalter. Monatsschr Kinderheilkd 2016; 164: $448-454$

\section{Bibliografie}

DOI http://dx.doi.org/10.1055/s-0042-117361 Pädiatrie up2date 2017; 12: 1-24

(C) Georg Thieme Verlag KG Stuttgart · New York ISSN 1611-6445 


\section{Punkte sammeln auf CME.thieme.de}

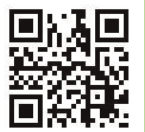

Diese Fortbildungseinheit ist 12 Monate online für die Teilnahme verfügbar.

Sollten Sie Fragen zur Online-Teilnahme haben, finden Sie unter cme.thieme.de|hilfe eine ausführliche Anleitung. Wir wünschen viel Erfolg beim Beantworten der Fragen!

Unter eref.thieme.de/ZZWHJNW oder über den QR-Code kommen Sie direkt zum Artikel zur Eingabe der Antworten.

VNR 2760512017152370195

\section{Frage 1}

Welche diagnostische Maßnahme führen Sie bei Regurgitation/ Spucken mit Zeichen einer Refluxerkrankung im Säuglingsalter als erste durch?

A Ösophagogastroduodenoskopie

B ösophageale pH-Metrie

C Breischluck mit Röntgenkontrastmittel

D ösophageale Impedanzmessung

E probatorische kuhmilchfreie Kost

\section{Frage 2}

Welche Aussage zur Laktoseintoleranz ist zutreffend?

A Eine Laktoseintoleranz führt in der Regel bereits im frühen Säuglingsalter zu klinischen Problemen.

B Die genetischen Polymorphismen im Laktase-Gen korrelieren klar mit den klinischen Symptomen.

C Die Diagnose erfolgt mit einer Laktosebelastung und anschließenden Blutzuckerkontrollen.

D Bei einer Laktoseintoleranz sind immer auch sekundäre Ursachen zu bedenken.

E Ein Auslassversuch mit Protokollierung der Symptome ist diagnostisch zu unsicher und sollte mit anderen Methoden überprüft werden.

\section{Frage 3}

Welche Aussage zur Diagnostik chronisch entzündlicher Darmerkrankungen trifft nicht zu?

A Bei Kindern jünger als 5 Jahre muss differenzialdiagnostisch an Immundefekterkrankungen gedacht werden.

B Infektiöse Darmerkrankungen müssen ausgeschlossen werden.

C Perianale Veränderungen sind Hinweise auf eine Colitis ulcerosa.

D In der bildgebenden Diagnostik sollte in der Regel eine Ösophagogastroduodenoskopie, eine lleokoloskopie mit Histologie und eine Bildgebung des Dünndarms erfolgen (z. B. Sonografie, MRT).

E Eine erhöhte Calprotectinkonzentration im Stuhl weist auf einen intestinalen Schleimhautschaden hin.

\section{Frage 4}

Welche Aussage zur Diagnostik einer Zöliakie trifft zu?

A Den Gliadin-IgG-Antikörpern kommt in der Diagnostik eine wichtige Rolle zu.

B Die Diagnose stützt sich auf die Transglutaminase-IgA-Antikörper, die Endomysium-Antikörper und die Positivität von HLA-DQ2 und/oder HLA-DQ8.

C Eine Endoskopie mit Gewinnung von Duodenalbiopsien ist obligat.

D Eine Bestimmung des Gesamt-IgA ist bei positiven Transglutaminase-IgG-Antikörpern nicht zwingend erforderlich.

E Bei Verdacht auf Zöliakie sollte unverzüglich mit einer glutenfreien Kost begonnen werden.

\section{Frage 5}

Welche Aussage zu Schluckstörungen im Kindesalter stimmt nicht?

A Ursache kann eine Achalasie sein.

B Ursache kann eine akute Gastroenteritis sein.

C Ursache kann eine eosinophile Ösophagitis sein.

D Ursache kann eine gastroösophageale Refluxerkrankung sein.

E In der Diagnostik kann in der Regel auf eine Ösophagoskopie nicht verzichtet werden.

\section{Frage 6}

Woran denken Sie primär bei einer Hämatochezie bei einem sonst gesunden jungen Säugling?

A Blutungsübel

B bakterielle/virale Enteritis

C Meckel-Divertikel

D Hämorrhoiden

E allergische Proktokolitis

Weitere Fragen auf der folgenden Seite ... 


\section{Punkte sammeln auf CME.thieme.de}

Fortsetzung ...

\section{Frage 7}

Was ist die wichtigste diagnostische Maßnahme bei Verdacht auf eine exokrine Pankreasinsuffizienz?
A Blutzuckertagesprofil
B Pankreaselastase im Stuhl
C Sekretin-Pankreozymin-Test
D Lipasebestimmung im Serum
E molekulargenetische Diagnostik einer zystischen Fibrose

\section{Frage 8}

Was ist eine wichtige Erkrankung in der Differenzialdiagnostik einer neonatalen Cholestase?
A akute Hepatitis A
B M. Wilson
C Autoimmunhepatitis
D Alpha-1-Antitrypsinmangel
E EBV-Infektion

\section{Frage 9}

Welche Aussage zur Gallengangsatresie trifft zu?

A Bei einer Gallengangsatresie sind die jungen Säuglinge innerhalb kurzer Zeit lebensbedrohlich erkrankt.

B Die Erkrankung liegt bereits zum Zeitpunkt der Geburt vor.

C Die Diagnose muss vor dem 60. Lebenstag gestellt werden, damit eine operative Korrektur (Hepatoportoenterostomie) noch sinnvoll ist.

D Die Erkrankung kann auch anikterisch verlaufen.

E Die Erkrankung kann mittels Laborparametern diagnostiziert werden.

\section{Frage 10}

Welche Antikörper sind in der Differenzialdiagnostik einer Autoimmunhepatitis im Jugendalter verzichtbar?
A ANA (antinukleäre Antikörper)
B SLA (soluble liver antibody)
C SMA (smooth muscle antibody)
D LKM (liver kidney microsome antibody)
E AMA (antimitochondriale Antikörper) 